\title{
Opportunistic Resilience Embedding (ORE): Toward Cost-efficient Resilient Virtual Networks
}

\author{
Rodrigo R Oliveira, Daniel S Marcon, Leonardo R Bays, Miguel C Neves, \\ Luciano P Gaspary, Marinho P Barcellos \\ Federal University of Rio Grande do Sul \\ Deep Medhi \\ University of Missouri-Kansas City
}

\begin{abstract}
Network Virtualization promotes the development of new architectures and protocols by enabling the creation of multiple virtual networks on top of the same physical substrate. One of its main advantages is the use of isolation to limit the scope of attacks - that is, avoiding traffic from one virtual network to interfere with the others. However, virtual networks are still vulnerable to disruptions on the underlying network. Particularly, high capacity physical links constitute good targets since they may be important for a large number of virtual networks.

Previous work protects virtual networks by setting aside backup resources. Although effective, this kind of solution tends to be expensive, as backup resources increase the cost to infrastructure providers and usually remain idle. This paper presents ORE (Opportunistic Resilience Embedding), a novel embedding approach for protecting virtual links against substrate network disruptions. ORE's design is two-fold: while a proactive strategy embeds each virtual link into multiple substrate paths in order to mitigate the initial impact of a disruption, a reactive one attempts to recover any capacity affected by an underlying disruption. Both strategies are modeled as optimization problems. Additionally, since the embedding problem is $\mathcal{N} \mathcal{P}$-Hard, ORE uses a Simulated Annealing-based meta-heuristic to solve it efficiently. Numerical results show that ORE can provide resilience to disruptions at a lower cost.
\end{abstract}

Keywords: Network Virtualization, Virtual Network Embedding, Resilience, Survivability, Multipath Embedding, Multiple Paths

Email addresses: \{ruas.oliveira, daniel.stefani, lrbays, mcneves, paschoal, marinho\}@inf .ufrgs.br (Rodrigo R Oliveira, Daniel S Marcon, Leonardo R Bays, Miguel C Neves, Luciano P Gaspary, Marinho P Barcellos), dmedhi@umkc.edu (Deep Medhi) 


\section{Introduction}

The Network Virtualization paradigm aims to simplify the development of new architectures and protocols. In particular, it allows networks to coexist over a shared physical infrastructure [1]. One of the main advantages of this paradigm is the use of isolation to limit the scope of attacks. This can be achieved by creating different, isolated virtual networks for each task or service so that traffic from one virtual network does not interfere with the others [2].

In this paradigm, the physical infrastructure (also referred to as the substrate network) may embed ${ }^{1}$ virtual networks in an on-demand manner [3]. To guarantee isolation, a substrate network administrator may perform admission control to each virtual network. In particular, it assigns shared physical resources to each virtual node and link of a virtual network. Hereafter, the term Virtual Network Embedding (VNE) will denote this process or the phase.

During VNE, several virtual networks are overlaid in specific regions of the substrate network [4]. From a security perspective, this characteristic makes virtual networks more vulnerable to disruptions in the physical infrastructure. In particular, VNE tends to increase the dependency on a small set of physical resources. Hence, a failure (or a successful attack) on a physical link can affect a large number of virtual networks.

Previous research tackled this problem by setting aside extra resources as backup $[5,6,7,8,9]$. This type of strategy may not be affordable since backup resources are not used for new allocations. Therefore, these approaches can reduce the ability of the network to handle new requests.

In this paper, we propose a novel embedding approach that optimistically improves the resilience of virtual networks without expending additional resources. Our approach is composed of two complementary optimization strategies, one proactive and the other reactive. The proactive strategy attempts to mitigate the initial impact of an attack by embedding each virtual link into multiple paths, thus preventing the virtual links from losing all their capacity. The reactive strategy aims at partially or fully recovering any capacity lost due to failures or compromised by attacks. To achieve this goal, the set of unaffected paths (if such paths exist) is used to opportunistically recover the compromised capacity. The design of Opportunistic Resilience Embedding (ORE) is inspired by known restoration techniques (i.e., those applied to traditional networks for improving survivability).

The first strategy requires solving the VNE problem. In a previous work [10], a mixed-integer programming (MIP) formulation of a variation of this strategy was presented. Yet, since the VNE problem is NP-Hard [11, 12, 13, 14], MIP models cannot scale to solve large problems. Hence, to achieve solutions within a reasonable timeframe, we use a Simulated Annealing-based metaheuristic. It runs efficiently and provides near-optimal solutions, converging towards a global optimum. The second strategy requires solving a max-flow problem over a set

\footnotetext{
${ }^{1}$ The terms embed, map and allocate will be used interchangeably.
} 
of pre-computed paths (i.e., those computed by the first strategy). We present a linear programming formulation to solve this problem.

The main contributions of this paper are summarized as follows:

- We present the design and implementation of a novel virtual network embedding algorithm, ORE. In contrast to the previous work, ORE does not make use of dedicated backup resources. Instead, ORE attempts to attain efficient resource utilization to the substrate network and resilience to the virtual links together. To our knowledge, ORE is the first approach to integrate embedding and restoration for virtual networks and analyze its advantages;

- ORE's strategies are modeled as optimizations problems. The proactive strategy is implemented with a Simulated Annealing-based algorithm, which achieves near-optimal solutions at a reasonable computing time. The reactive strategy is modeled as a linear programming (LP) problem, which is solved optimally and efficiently;

- Through simulation studies, we measured benefits of our approach. Based on our experimental studies, we found that ORE can reduce both bandwidth loss and severity of disruptions by increasing the number of paths used per virtual link. Further, when compared to backup-oriented schemes, ORE achieves a higher acceptance rate.

The rest of the paper is organized as follows: Section 2 introduces preliminary concepts and assumptions that will be used throughout the paper. The proposed solution, namely ORE, is described in Sections 3 and 4; it first provides an overview of the ORE's design ( $\$ 3$ ), followed by the implementation of both the proactive and the reactive strategies $(\S 4)$. Section 5 discusses numerical results, Section 6 reviews related work, and Section 7 concludes this paper.

\section{Preliminaries}

\subsection{Virtual Network Embedding Problem}

The Virtual Network Embedding (VNE) problem emerges from the interaction between virtual networks and the underlying substrate network. In short, it consists of an efficient allocation of virtual network topologies on top of the substrate network. Efficiency comes from the goal of maximization or minimization of an objective as defined by the owner of the substrate network, subject to satisfying demands imposed by an SLA agreed upon between the owners of the virtual networks and the substrate network provider. Depending on the requirements and the purpose of the virtual networks, the problem is to be solved either in real-time, or offline ahead of time. If virtual network requests arrive on-demand without any prior knowledge, they are to be allocated in real-time or near real-time; on the other hand, if demand is known beforehand for a future 


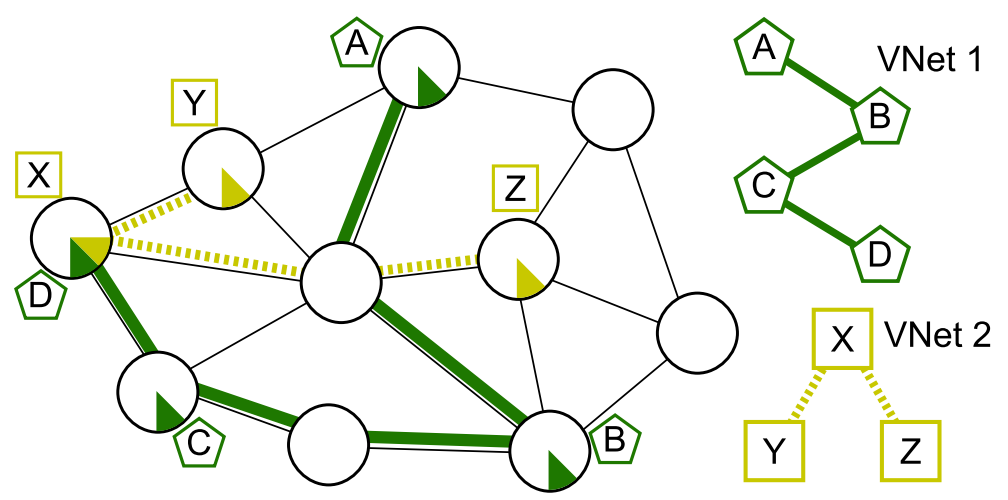

*Color and line styles are used to facilitate the identification of different virtual networks

(a) Two virtual networks mapped into a substrate network. Each virtual node is placed into a substrate node, consuming a fraction of the substrate node's resources.

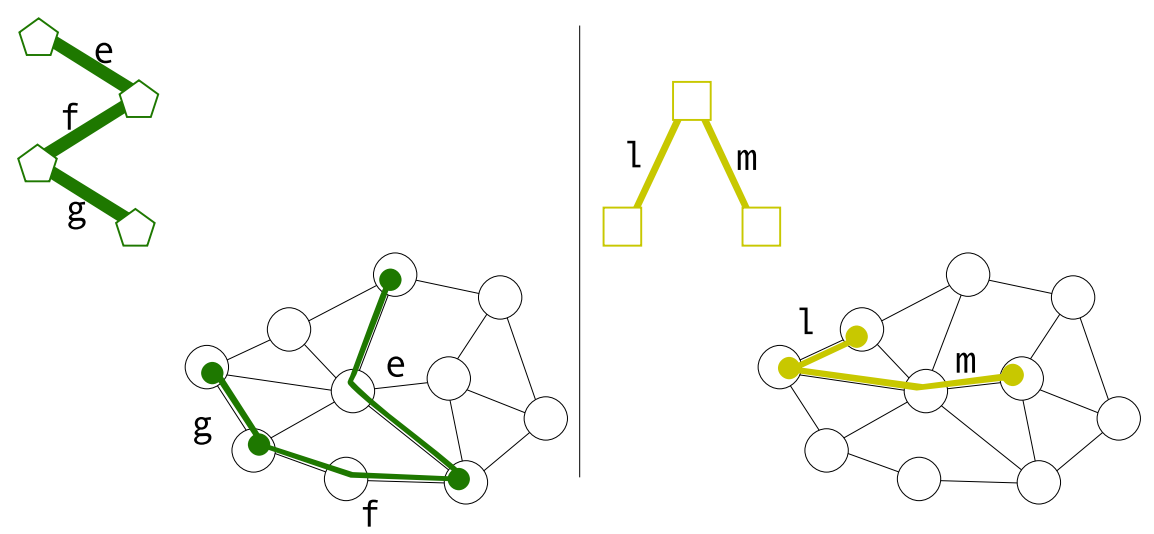

(b) Each virtual link is mapped into a substrate link or a substrate path. It is noteworthy that the selected substrate link/path should connect the endpoints of the virtual link.

Figure 1: Illustration of two virtual networks embedded in a substrate network, with both the unified and the separate representations of the resulting mapping. Colors and line styles are used to facilitate the identification of different virtual networks.

request, the problem can be solved offline ahead of time so that virtual networks can be allocated.

In general, each virtual network is composed of a virtual topology (interconnected by virtual routers and virtual links) and a variety of requirements. The substrate network is composed of substrate routers, which support a virtualization technology, interconnected by substrate links. Both substrate routers and substrate links have attributes and constraints (e.g., router CPU capacity, link bandwidth capacity) that dictate which features are available to the overlay 
virtual networks [15]. Therefore, virtual networks are free to implement potentially arbitrary services, as long as the substrate network is capable of offering the necessary features.

In order to match virtual requirements with substrate capabilities and achieve optimal utilization of resources, the substrate network administrator invokes an allocation process. This process consists of selecting feasible substrate devices and assigning shares of their resources to the overlaid virtual networks $[16,13$, 17]. Thus, each virtual node is mapped to a substrate node that supports all requirements; likewise, each virtual link is mapped to a substrate link or a continuous, loop-free substrate path that supports all requirements. Additionally, the endpoints of the substrate link (or path) selected to embed a virtual link should be the substrate nodes embedding the endpoints of the virtual link.

Figure 1 exemplifies possible mappings of two virtual networks into a substrate network. Note that the example is not exhaustive in terms of all possible mappings; rather, it is meant to illustrate a VNE scenario. For simplicity, this figure represents resources used by slices on the substrate nodes. Slices could represent a fraction of the node's processing power, packet processing throughput, number of entries in the routing table, etc. Although the figure does not explicitly illustrate link requirements, they are also taken into consideration.

Figure 1(a) shows the overall, unified mapping and resource allocation, highlighting the virtual node embedding, whereas Figure 1(b) shows each individual mapping and highlights the virtual link embedding. In Figure 1(a), VNet 1 is embedded into four substrate nodes, one for each virtual node: A, B, C, and D. VNet 2 is embedded in the same network. Nodes X, Y, and Z consume three different physical nodes. Nodes D and X are collocated on the same physical node. This is possible if no restriction is imposed (e.g., there is enough computational capacity).

Figure 1(b) shows that the virtual links 'e' (A-B) and ' $\mathrm{f}$ ' (B-C) are embedded into paths between the nodes, each using two physical links. The virtual link ' $\mathrm{g}$ ' (C-D) is embedded into a single substrate link. Similarly, the virtual link 'l' $(\mathrm{X}-\mathrm{Y})$ is embedded into a single substrate link while virtual link ' $\mathrm{m}$ ' (X-Z) is embedded into two substrate links (the path between $\mathrm{X}$ and $\mathrm{Z}$ ).

\subsection{Threats}

In this work, we focus on the disruption of the communication between virtual nodes caused by attacks (or failures) compromising parts of the substrate network. Disruptions may happen either at physical routers or physical links. In particular, an attack to a device may occur by obtaining physical access, or by exploiting vulnerabilities. The former happens when an attacker identifies the location of an optical fiber or a router and interrupts these devices, e.g., strategically planned fiber cuts have been known to affect thousands of customers ${ }^{2}$. The latter occurs when an attacker exploits some vulnerability on the control software or protocol to cause denial of service on a device. In this paper, we

\footnotetext{
${ }^{2}$ http://goo.gl/t4VYhW, goo.gl/JGx0ph
} 
assume that disruptions occur on a single router or link at a time. More specifically, we consider the worst case disruption that corresponds to a failure or an attack compromising the node with the most aggregated bandwidth embedded on its incident links. To model attacks, we consider that the attacker might (partially) infer the topology, identifying the most critical elements.

\subsection{Multipath Concerns}

Two primary concerns exist when splitting the capacity of a virtual link over multiple paths. First, the selected paths may present different propagation delays, which aggravates out-of-order delivery and may hurt application performance. Second, each path requires additional resources (table entries) on routers and periodic maintenance (e.g., availability checks).

Addressing out-of-order delivery. In IP networks, this problem is generally prevented by multiplexing network traffic at flow-level granularity. This is achieved by assigning each flow to a specific path based on its "flow-id", that is, five fields in the packet header (namely the src/dst IP addresses, the transport protocol, and the src/dst ports) [18]. To utilize such techniques, a virtual network environment would need to be able to identify to which flow each packet belongs. This should be straightforward, while IP-based virtual networks require no modification, non-IP virtual networks would only need to inform which bits in the packet header should be used to identify a flow [14].

Our algorithms assume flow-based traffic splitting so that differential delay does not became an issue. However, it is important to emphasize that other techniques, such as packet-based and flowlet-based splitting [18], could be used instead. In a deployment, routers should be configured with the desired splitting technique in order to behave accordingly.

Limiting overhead. To tackle the second problem, the number of paths should be kept as low as possible, thus reducing the overhead caused by additional table entries and control messages [19]. A practical approach for embedding a virtual link into multiple paths can be achieved by using a multicommodity flow formulation. Although such approaches yield good solutions, they offer little control over the number of distinct paths used for embedding [19]. Therefore, any solution for assigning virtual links to multiple paths should be upper bounded, thus allowing better control over the outcome of the embedding phase.

\section{ORE Design Overview}

In this section, we present an overview of the design of ORE (Opportunistic Resilience Embedding). The goal of ORE is to offer an embedding algorithm with efficient resource utilization and opportunistic protection jointly to virtual links against substrate network disruptions. The key idea in ORE is to use the substrate network diversity and unused capacity to proactively and reactively protect against these disruptions. Toward this end, ORE defines two complementary strategies, one proactive and the other reactive. 


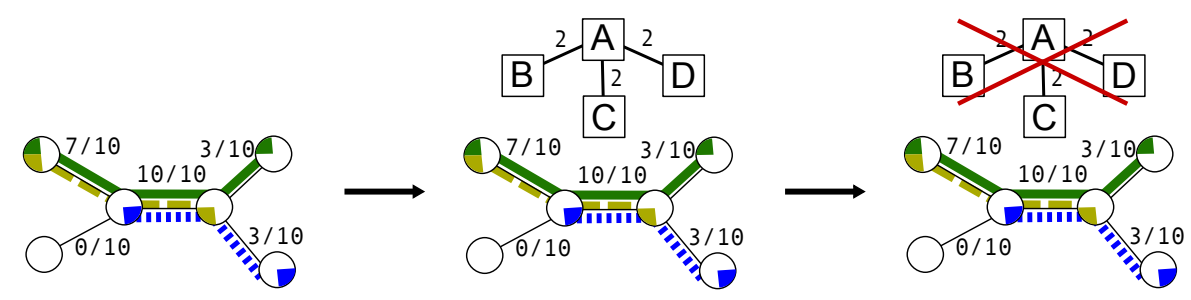

Figure 2: Rejection of a VNR due to the formation of a bottleneck. Colors and line styles are used to facilitate the identification of different virtual networks.

\subsection{Joint efficient embedding and opportunistic protection}

Efficient embedding. As mentioned earlier, in a network virtualization environment, we consider the case when virtual network requests are received on-demand by the substrate provider. This implies that, in the long-run, the allocation and deallocation of resources may lead to fragmentation. Such fragmentation, in turn, can cause a set of substrate links to become saturated. When the VNE algorithm does not consider such scenarios, virtual network requests can be rejected even if the substrate has a sufficient aggregated residual capacity.

In an attempt to provide better utilization of substrate network resources, a common optimization objective in VNE algorithms is to perform load balancing. This technique tends to avoid the creation of bottleneck links, thus potentially increasing the VNR acceptance rate [16]. Figure 2 illustrates the formation of a bottleneck due to saturation-unaware resource allocation algorithms. The figure shows a substrate network on which several virtual networks are embedded. In this example, most of the capacity of virtual links is being allocated to a single substrate link that becomes saturated. As a result, a virtual network request that needs this link is rejected. If saturation was avoided by using the free node/link, then the request could be accepted.

To provide load balancing, ORE takes advantage on a classic Traffic Engineering (TE) function available in the literature [20]. More specifically, ORE employs a piecewise linear increasing convex function of link utilization with the same values defined in the literature $[21,22]$.ORE uses this function as the objective function when performing the VNE process. In particular, ORE gives preference to lower cost substrate links when composing the paths that embed virtual links. This way, substrate links with high utilization are less likely to be selected. Let $\Phi($.$) denote a piecewise linear increasing convex function of link$ utilization, as defined in [21]. Accordingly $\Phi\left(\right.$ util $\left.\left(e^{S}\right)\right)$, henceforth denoted as $\phi_{e^{S}}$, yields the current cost of a given substrate link $e^{S} \in E^{S}$.

Mitigating disruptions. Opportunistic protection is provided by splitting the capacity of a virtual link on to multiple paths. During the VNE phase, each virtual link is embedded into multiple, distinct substrate paths, and its bandwidth demand is distributed among the capacity of these paths. Hence, if a disruption occurs, only a subset of the paths of each virtual link would be affected. If the strategy is successful, virtual links will remain operational - at 


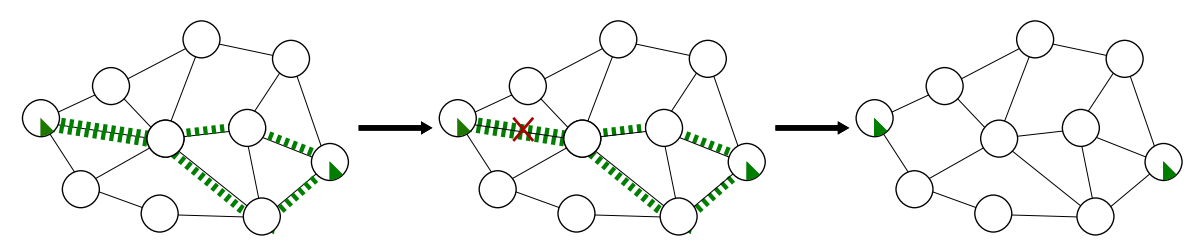

Figure 3: Inefficient resilient multipath embedding.

a lower capacity - after disruptions.

The effectiveness of this strategy depends on the paths chosen in the embedding phase. More specifically, unless the selected paths are sufficiently different, a virtual link will still be vulnerable when a single substrate link embeds most - or all - of the virtual link capacity. Figure 3 shows an example of a virtual link with its capacity split into two paths. This mapping is valid, but does not benefit from a resiliency perspective. When a disruption occurs on a shared substrate link, the virtual link becomes unresponsive. The issue, known as the shared risk link group (SRLG), has been studied before [23, 24, 25]. Therefore, the embedding phase attempts to avoid path similarity, whenever possible, when selecting substrate paths to embed virtual links.

\subsection{Opportunistically reacting to disruptions}

The objective of the second strategy is to restore some of the capacity of a virtual link from being compromised by disruptions on substrate routers and links (i.e., those utilized to embed the virtual link). Towards this end, ORE devises a restoration technique that uses the information from the embedding to react against disruptions. Note that restoration is best-effort in nature and may have a slower recovery time than backup-oriented techniques.

For restoration, ORE relies on the residual capacity of the substrate network to try to recover the compromised capacity of a virtual link. More specifically, after a disruption event occurs, a subset of the virtual links will be either partially or fully compromised. A virtual link is partially compromised when some of its embedding substrate paths remain operational; these paths are called active paths. Hence, if a virtual link has active paths, the strategy tries to increase the reserved bandwidth of these paths to compensate the lost capacity. It is noteworthy that fully compromised virtual links do not have active paths and thus cannot be restored by this technique. As will be discussed in the evaluation section, given enough diversity, this situation becomes unlikely for single node or link failures.

An example of the reactive strategy is shown in Figure 4. Two virtual networks, each with two virtual nodes and one virtual link, are embedded on to the same substrate network. The virtual link of the first network (green, slashed lines) is embedded on three substrate paths; similarily, the virtual link of the second network (blue, dotted lines) is embedded on to two substrate paths. In the example, when an embedding substrate link " $w$ " becomes unresponsive, the 


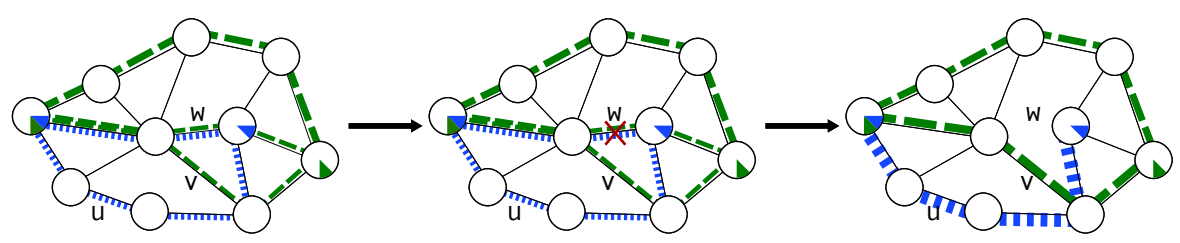

Figure 4: ORE approach for surviving substrate network disruptions. Colors and line styles are used to facilitate the identification of different virtual networks.

capacity of any available active path on both virtual networks is increased (e.g., substrate paths using links " $u$ " and " $v$ ").

\section{ORE Implementation}

We now present the implementation of the two strategies used by ORE. As previously stated, the proactive strategy ( $\$ 4.1)$ is used during embedding, while the reactive one is applied after a disruption $(\S 4.2)$. For ease of reference, the symbols used in this section are listed in Table 1.

\subsection{VNE with Multipath and Opportunistic Resilience}

This strategy attempts to embed virtual network requests on a given substrate network with the goal of providing resilience for virtual networks and efficient allocation of substrate resources. In this proactive strategy, resilience is achieved by wisely selecting paths that will be used for virtual link embedding. More precisely, in this strategy, opportunistic resilience refers to the path selection step that avoids path similarity; in other words, opportunistic resilience minimizes the SRLG.

\subsubsection{Problem Model}

The model described here is in accordance with previous work dealing with the VNE problem (e.g., $[12,13,14])$. Virtual nodes and links are embedded on substrate nodes and paths, respectively. Each virtual node is embedded in a single substrate node while each virtual link may be embedded on to one or more paths of the substrate network. Additionally, virtual nodes of the same virtual network must be embedded on distinct substrate nodes. The embedding of virtual networks is performed on-demand (i.e., online), as requests arrive. Hence, it is not possible to determine beforehand which virtual networks should be embedded.

Network. A virtual network request or a substrate network can be represented as a weighted graph with a list of attributes. Nodes, edges, and the connections between them correspond to the network topology, while the list of attributes represents virtual network requirements or substrate network features. Tuple $G\langle N, E, A\rangle$ denotes a weighted, directed or undirected graph with a set $N$ of nodes, a set $E$ of edges (links), and a set $A$ of node or edge attributes. 
Table 1: Symbols used in this article.

\begin{tabular}{|c|c|}
\hline Symbol & Description \\
\hline \multicolumn{2}{|r|}{ Mathematical Symbols } \\
\hline$\mapsto$ & Maps to: represents mapping between sets or between elements. \\
\hline$\leftarrow$ & $\begin{array}{l}\text { Assignment: leftside operand (variable or set) is assigned the value of the } \\
\text { rightside operand (variable, set, or constant). }\end{array}$ \\
\hline$\Rightarrow$ & Implication: $A \Rightarrow B$ means, if $\mathrm{A}$ is true, then $\mathrm{B}$ has to be true. \\
\hline iff,$\Longleftrightarrow$ & $\begin{array}{l}\text { If, and only if; double implication: } A \Longleftrightarrow B \text { means that A is only true if } \\
\mathrm{B} \text { is true, and vice-versa. }\end{array}$ \\
\hline \multicolumn{2}{|r|}{ Sets, Variables, and Constants } \\
\hline$G\langle N, E, A\rangle$ & $\begin{array}{l}\text { Weighted, directed or undirected graph representing a substrate or a virtual } \\
\text { network. }\end{array}$ \\
\hline$G^{S}, G^{V}$ & 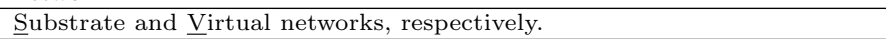 \\
\hline$N^{S}, N^{V}$ & $\overline{\text { Set of substrate or virtual nodes. }}$ \\
\hline$E^{S}, E^{V}$ & Set of substrate or virtual edges (links). \\
\hline$P^{S}$ & Set of substrate continuous, loop-free paths. \\
\hline$|P|$ & Maximum number of paths used to embed a virtual link. \\
\hline$A^{S}, A^{V}$ & $\begin{array}{l}\text { Set of substrate or virtual attributes (virtual network requirements or sub- } \\
\text { strate network features). }\end{array}$ \\
\hline$\kappa$ & $\begin{array}{l}\text { Constant penalty used in cost(.) function for invalid solutions. Holds the } \\
\text { aggregated cost of all substrate links when saturated ( } 100 \% \text { utilization). }\end{array}$ \\
\hline$\pi_{n S} S$ & Probability of choosing a substrate node. \\
\hline$\pi_{e} S$ & Probability of choosing a substrate link. \\
\hline$x_{e V, p} S \%$ & $\begin{array}{l}\text { Fraction of bandwidth demand of a virtual link } e^{V} \text {, allocated in a given } \\
\text { path } p^{S} \text {. }\end{array}$ \\
\hline \multicolumn{2}{|r|}{ Operators and Functions } \\
\hline $\mathcal{M}^{\mathcal{N}}: N^{V} \mapsto N^{S}$ & Function mapping nodes of set $N^{V}$ into nodes of set $N^{S}$. \\
\hline $\mathcal{M}^{\mathcal{E}}: E^{V} \mapsto P^{S}$ & Function mapping edges of set $E^{V}$ into paths of set $P^{S}$ \\
\hline $\mathcal{M}^{\mathcal{E}}{ }_{i}: E^{V} \mapsto P^{S}$ & $\begin{array}{l}\text { Function mapping edges of set } E^{V} \text { into multiple paths of set } P^{S} \text {; paths are } \\
\text { indexed by } i \in|P|\end{array}$ \\
\hline$c()$. & $\begin{array}{l}\text { Required CPU demand of a virtual node }\left(c\left(n^{V}\right) \mid n^{V} \in N^{V}\right) \text {, or current } \\
\text { residual capacity of a substrate node }\left(c\left(n^{S}\right) \mid n^{S} \in N^{S}\right)\end{array}$ \\
\hline$b()$. & $\begin{array}{l}\text { Required bandwidth demand of a virtual link }\left(b\left(e^{V}\right) \mid e^{V} \in E^{V}\right) \text {, or cur- } \\
\text { rent residual capacity of a substrate link }\left(b\left(e^{S}\right) \mid e^{S} \in E^{S}\right)\end{array}$ \\
\hline alloc $()$. & Amount of resources allocated in a substrate resource \\
\hline $\operatorname{resid}()$. & Amount of available resources of a substrate resource \\
\hline util(.) & Fraction (within $[0,1]$ ) of utilization of a given substrate resource \\
\hline$\Phi()$. & $\begin{array}{l}\text { Link saturation measurement function: } \Phi\left(\text { util }\left(e^{S}\right)\right) \text { returns a cost which } \\
\text { is exponentially proportional to link utilization. }\end{array}$ \\
\hline$\phi_{e} S$ & Same as $\Phi\left(\operatorname{util}\left(e^{S}\right)\right)$ \\
\hline $\operatorname{cost}()$. & $\begin{array}{l}\text { Cost function of Simulated Annealing: } \operatorname{cost}(S) \text { evaluates the total link } \\
\text { saturation of the substrate network of a potential solution " } S \text { ". }\end{array}$ \\
\hline initial_solution() & $\begin{array}{l}\text { Solution generation function of Simulated Annealing; creates an initial so- } \\
\text { lution " } S \text { " using a greedy algorithm. }\end{array}$ \\
\hline neighbor(.) & $\begin{array}{l}\text { Neighbor function of Simulated Annealing: neighbor }(S) \text { creates a solution } \\
\text { " } S \text { " which is similar to a previously generated solution " } S \text { " }\end{array}$ \\
\hline
\end{tabular}

Superscripts $V$ and $S$ are used, when necessary, to distinguish between a virtual or a substrate network; e.g., $G^{V}\left\langle N^{V}, E^{V}, A^{V}\right\rangle$ and $G^{S}\left\langle N^{S}, E^{S}, A^{S}\right\rangle$ represent a virtual network and a substrate network, respectively,.

Attributes. Capacities and requirements can be queried from a virtual or substrate network. Queries from a virtual network denote a requested amount of the given attribute, while queries from a substrate network denote residual capacity. Here, we focus on the CPU capacity of routers and the bandwidth of links; thus, attributes are represented as follows: $c\left(n^{S \mid V}\right)$ is the CPU capacity of node $n^{S \mid V} \in N^{S \mid V}$, and $b\left(e^{S \mid V}\right)$ is the bandwidth of link $e^{S \mid V} \in E^{S \mid V}$. 
Mapping. The number of paths in each virtual link is denoted by $|P|$, where set $P$ enumerates the paths from 1 to $|P|$. Binary variables $\mathcal{M}\left(n^{V}, n^{S}\right)$ and $\mathcal{P}\left(e^{V}, e^{S}\right)$ denote node and link assignments, respectively. $\mathcal{M}\left(n^{V}, n^{S}\right)$ indicates if a virtual node $n^{V} \in N^{V}$ is mapped into substrate node $n^{S} \in N^{S}$, whereas $\mathcal{P}\left(e^{V}, e^{S}\right)$ indicates if link $e^{S} \in E^{S}$ is used in the mapping of virtual link $e^{V} \in$ $E^{V}$. In addition, functions alloc(.) and resid(.) denote the amount of allocated and residual resources of a given element (e.g., alloc $\left(e^{S}\right)$ indicates the amount of bandwidth already allocated on link $\left.e^{S}\right)$.

\subsubsection{Embedding Metaheuristics using SA.}

Since the VNE problem is an $\mathcal{N} \mathcal{P}$-Hard problem [11], an optimal result is difficult to generate for large networks in near real-time. To solve this issue efficiently, ORE develops a metaheuristic based on Simulated Annealing (SA). The solution attained by this metaheuristic algorithm is sub-optimal; however, the advantage is that we can obtain a solution quickly for use in real-time or near real-time. The SA pseudo-code is described in Algorithm 1. It starts by using a greedy algorithm to create an initial mapping that may or may not be a valid solution. Then, instead of exploring the entire solution space for the best mapping, SA iteratively generates possible mappings until a maximum number of steps $K$ (Line 8) is reached.

In each step $k$, SA tries to improve the current solution $S$ by creating several $(R)$ neighbors (similar) solutions $S^{\prime}$ (Line 10). In addition, SA uses the concept of temperature $(T)$ and cooling factor $(0<c<1)$ to escape local minima. This works by selecting slightly worse solutions with the probability being directly proportional to the temperature $\left(e^{-\left[\operatorname{cost}\left(S^{\prime}\right)-\operatorname{cost}(S)\right] / T}\right)$. Since the temperature is decreased at each step $(k)$, this action is less likely to happen in the last few steps of SA when a solution is close to optimal. Finally, when SA terminates, the best overall attained solution $S^{*}$ is returned.

The parameters of SA, namely the number of steps $(K)$, the number of neighbors per step $(R)$, the temperature $(T)$, and the cooling factor $(c)$, were tuned during experimentation. Therefore, their discussion is deferred to Section 5. Next, we discuss the key components of Simulated Annealing: (i) the cost function that measures the quality of a solution; (ii) the initial solution, which is iteratively modified in an attempt to converge to the global optimum; and (iii) the neighbor function, used to modify a solution.

Cost function. The quality of attained solutions is compared by measuring link saturation over the network. The objective is to achieve better resource utilization by avoiding bottlenecks. This is performed by employing the traffic engineering function $\Phi$, previously described in Section 3.1. However, this function cannot be used "as-is" in this metaheuristic, since the algorithm should differentiate valid solutions from invalid ones. During the execution of a metaheuristic, several solutions are obtained at random. Although valid solutions with worse embeddings have higher costs, invalid solutions may not. For example, consider a case where an empty substrate has to embed one virtual network with a single virtual link; further, suppose two solutions are found, one which the virtual link is properly embedded on a number of paths, and another where 


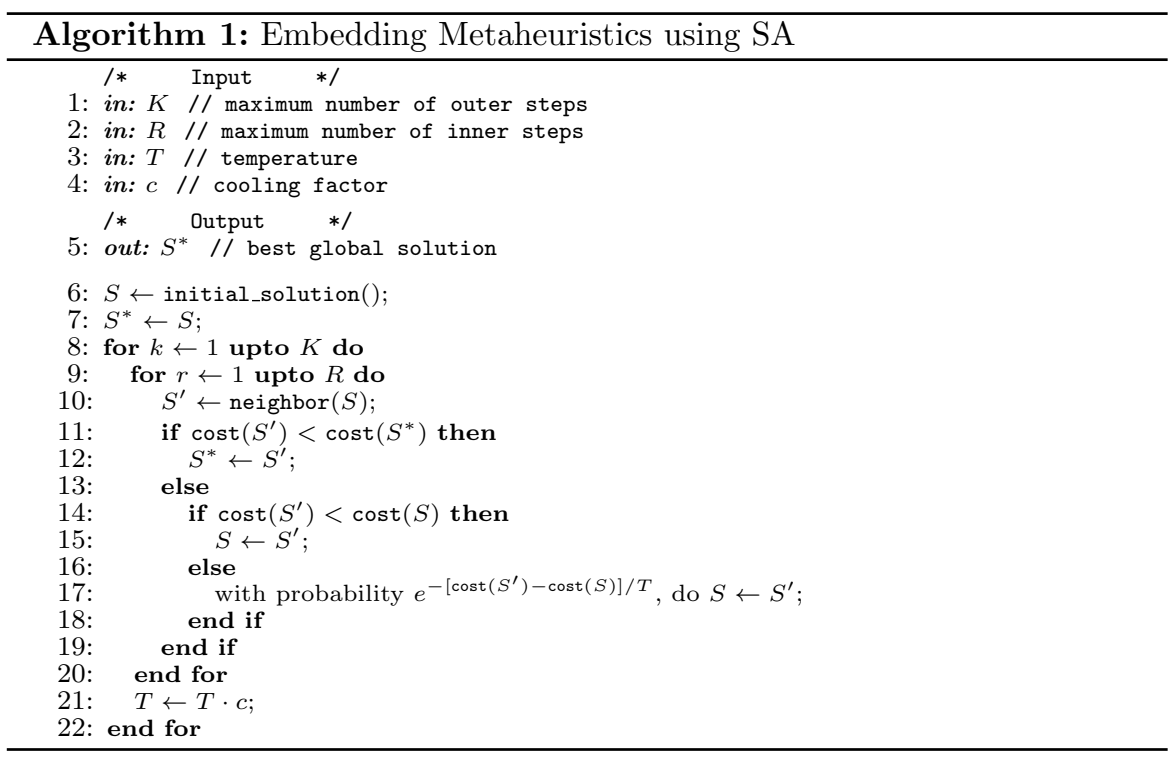

no path has been selected to embed this virtual link. Clearly, the first solution is valid while the second is invalid. However, by measuring only link saturation, the second solution will have a lower cost - in fact, the cost will be zero (i.e., the minimum value) because no substrate link is saturated. To solve this problem, the cost function adds a penalty $\kappa$ to all invalid solutions; $\kappa$ is equal to the worst possible solution. This value is calculated by multiplying the number of substrate links (i.e., $\left|E^{S}\right|$ ) by the value of a fully saturated link [i.e., $\left.\Phi(1)\right]$. The cost function is given by the following expression:

$$
\operatorname{cost}= \begin{cases}\sum_{e^{S} \in E^{S}} \phi_{e^{S}}, & \text { if solution is valid } \\ \sum_{e^{S} \in E^{S}} \phi_{e^{S}}+\kappa, & \text { if solution is invalid }\end{cases}
$$

where $\kappa=\left|E^{S}\right| \cdot \Phi(1)$.

Initial solution. A solution is obtained by a greedy algorithm (which is latter improved in the neighbor function). The algorithm is composed of three steps: the node embedding phase, the link embedding phase, and the capacity assignment phase. The entire procedure is described by Algorithm 2 . In the node embedding phase, each virtual node is randomly placed on substrate nodes with enough capacity to hold them. Since virtual nodes from the same request cannot share the same substrate node, the probability of choosing a substrate node $\left(\pi_{n}\right)$ is inversely proportional to the number of virtual nodes 
already allocated:

$$
\pi_{n^{S}}=\frac{1}{1+\sum_{\forall n^{V} \in N^{V}} \mathcal{M}^{\mathcal{N}}: n^{V} \mapsto n^{S}},
$$

where $\pi_{n^{S}}$ is normalized so that the sum of all probabilities adds to 1 (i.e., $\left.\sum_{\forall n^{S} \in V} \pi_{n S}=1\right)$.

The link embedding phase uses a depth-first search to allocate the paths of each virtual link. However, instead of selecting edges deterministically, it uses a probability $\pi_{e}$ based on two metrics: (a) link utilization; and (b) path similarity. The higher the value of one of these metrics (or both), the lower the probability to select the link when constructing a path. In other words, $\pi_{e}$ ensures load balancing and resilience by avoiding saturated or SRLG links when selecting paths using depth-first search. Probability $\pi_{e}$ is calculated as follows:

$$
\pi_{e^{S}}=\frac{1}{1+\operatorname{alloc}\left(e^{S}\right)}+\frac{1}{1+\sum_{\forall i \in|P|} \mathcal{M}^{\mathcal{E}}{ }_{i}: e^{V} \mapsto p^{S}}
$$

where $e^{S} \in p^{S}$.

In the first term of (3), function alloc(.) denotes how much bandwidth is already allocated, thus guiding the solution to less saturated links. The second term of (3) indicates how many paths of the same virtual link are using the given substrate link; hence, it penalizes links that represent an SRLG. Similar to $\pi_{n^{S}}$, this probability is normalized for all neighboring links (i.e., $\sum_{\forall u^{S} \in N^{S}} \pi_{e^{S}}=$ $\left.1, \forall v^{S} \in N^{S} \mid\left(u^{S}, v^{S}\right) \in E^{S}\right)$.

Finally, the capacity assignment phase consists of distributing the bandwidth demand of a virtual link among its embedding paths. In this phase, each path will receive a fraction proportional to its residual bandwidth, as follows:

$$
\begin{array}{r}
x_{e^{V}, p^{S}} \%=\frac{\operatorname{resid}\left(p^{S}\right)}{\sum_{\forall q^{S} \in P^{S} \mid \mathcal{M}^{\mathcal{E}}: e^{V} \mapsto q^{S}} \operatorname{resid}\left(q^{S}\right)}, \\
\forall p^{S} \in P^{S} \mid \mathcal{M}^{\mathcal{E}}: e^{V} \mapsto p^{S} .
\end{array}
$$

In (4), function resid(.) indicates how much residual capacity is available on a given path; this is calculated by obtaining the minimum residual capacity among the links composing the path. Notice that before assigning capacity, it is necessary to check if the path has sufficient bandwidth. Thus, the amount of bandwidth allocated to a path $p$ is upper bounded by the following expression: $\min \left(\operatorname{resid}(p), x_{p} \% \cdot b\left(e^{V}\right)\right)$.

Neighborhood and local improvement. SA uses neighbors in an attempt to improve the current solution or escape a local minimum. A neighbor is generated by applying simple modifications to the current solution, resulting 


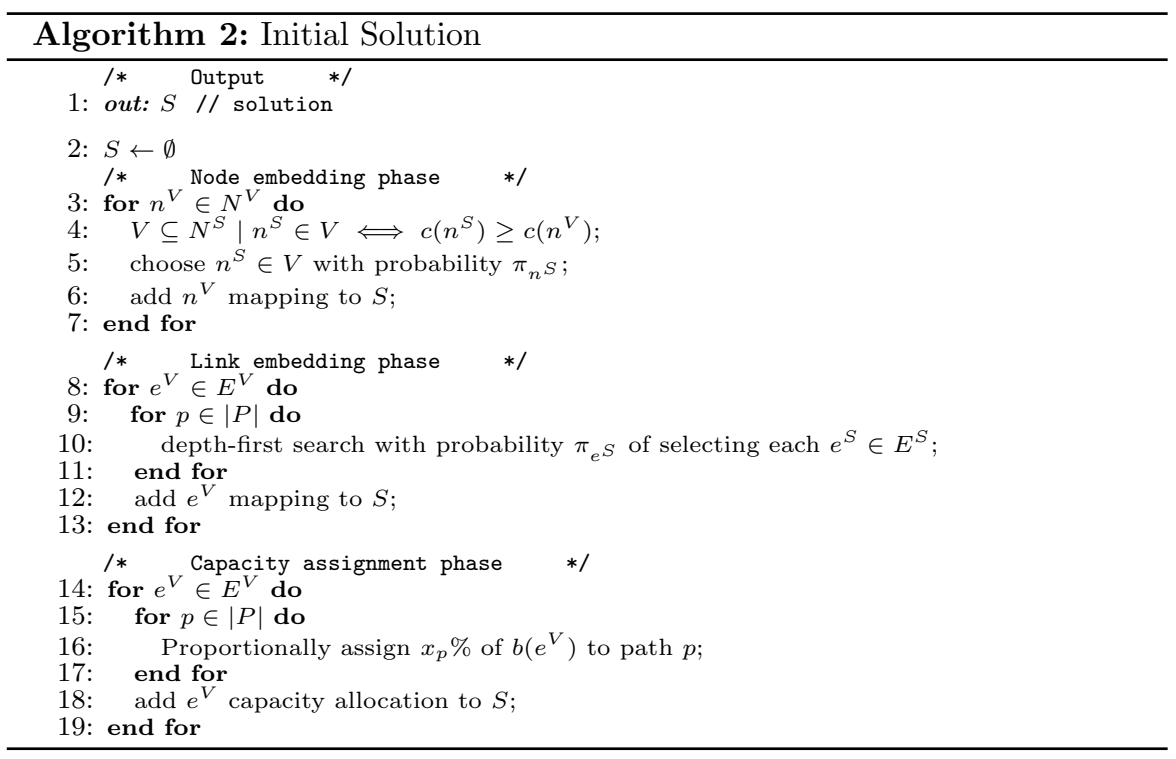

in a similar (potentially better) solution. This procedure is composed of two phases: the first selects a random solution from the available neighborhood, while the second repeatedly improves this solution. Both phases execute the same key steps, with a minor difference on the probability of selecting a new neighbor. Algorithm 3 provides a pseudo-code of the entire procedure.

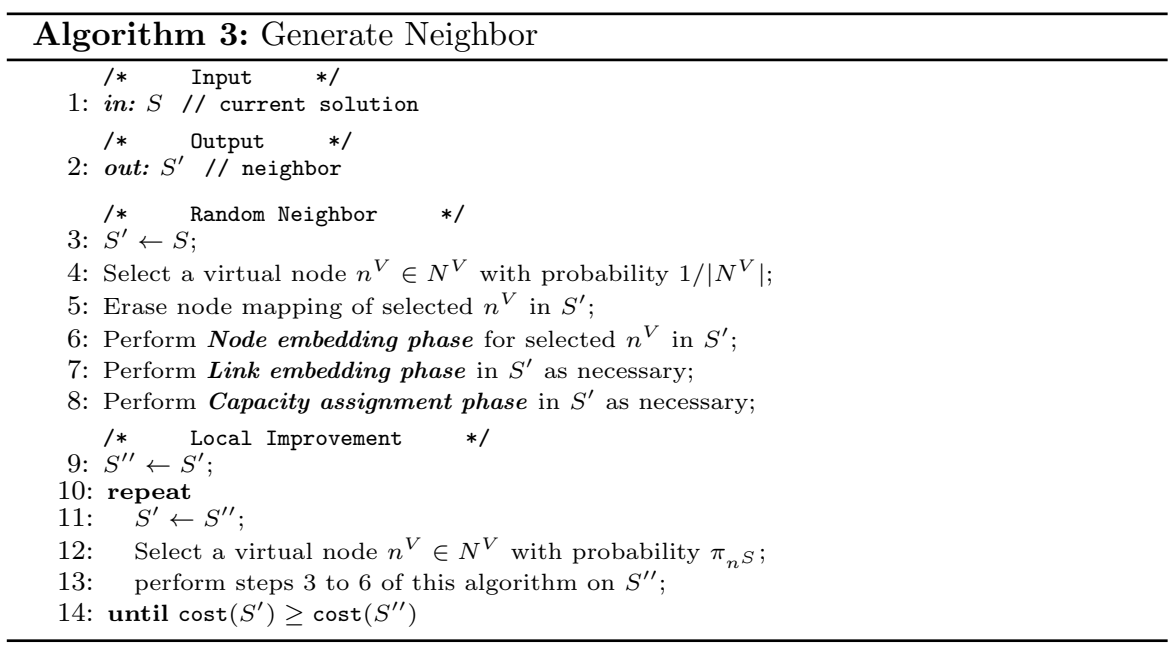

The algorithm consists of moving a randomly selected virtual node to another substrate node and recalculating paths affected by this action. In the first 
phase of the algorithm, a virtual node $n^{V}$ is selected uniformly from the set of all virtual nodes $N^{V}$. This allows a solution to escape a local minimum by generating a similar solution (this initial solution is potentially worse). Then, in the second phase, the new solution is improved incrementally by reallocating nodes with probability $\pi_{n^{S}}$ [see (2)], thus attempting to converge to another potentially better - local minimum.

The node/link embedding and capacity assignment phases of this procedure are performed similar to the ones described in the previous procedure (Algorithm 2). The sole difference being that the node embedding phase is performed only on the selected nodes; and accordingly, the link embedding and capacity assignment phases are performed only on the virtual links that were broken by the reallocation of the virtual node.

\subsection{Opportunistic Capacity Recovery}

The previous subsection considers the networks in normal operation, that is, prior to disruptions. This subsection, in contrast, looks at networks after disruptions. In particular, it attempts to respond to two questions: what happens to the virtual networks when a disruption in the substrate occurs? And are such events handled?

After a disruption occurs, a virtual link may enter three different states: unaffected, fully compromised, or partially compromised. Recall that virtual links are embedded into one or multiple substrate paths in the network (§ 3.1). Thus, a virtual link is unaffected when the disruption does not occur in any of the substrate links used by the paths embedding the virtual link. Conversely, if the disruption occurs in any substrate link used by the virtual link, then the virtual link becomes compromised (either fully or partially).

A virtual link is fully compromised if it has no more paths left. If the paths of the virtual link are a shared risk link group (SRLG), then this may happen even if only a single substrate link is disrupted (see, Fig. 3 in $\S 3.1$ ). Finally, a virtual link is only partially compromised if one or more paths remain operational - from this point onwards, these paths will be referred as active paths.

A key contribution of this work is the opportunistic recovery mechanism. Instead of allocating backup resources, the capacity compromised by a disruption is recovered over the residual bandwidth along the set of active paths. We next present the formulation of this strategy as a linear programming model.

In this LP model, a set of variables keeps track of how much bandwidth is being requested, while input sets define which substrate links will be used and how much bandwidth was lost. The objective of the model is to allocate all residual capacity of the active paths. Constraints are used to avoid wasting resources.

Variable. $Q_{p, e^{V}} \in \mathbb{R}$ within $[0,1]$ is used to represent the fraction of the virtual link $e^{V} \in \dot{E}^{V}$ capacity that will be recovered in active path $p$. For example, $Q_{p, e^{V}}=0.1$ means 10 percent of the capacity of $e^{V}$ is being reassigned to active path $p$. Suppose that $e^{V}$ has a nominal capacity of $10 \mathrm{Mbps}$; this means that an additional $1 \mathrm{Mbps}$ will be reserved on each substrate link along active path $p$. 


\section{Input.}

- set $\hat{E}^{S} \subset E^{S}$ defines the substrate links that remain available after a disruption event; since compromised links are unaccessible, they cannot be used to reallocate any bandwidth;

- set $\dot{E}^{V} \subseteq E^{V}$ enumerates the virtual links affected by the disruption; all other virtual links are omitted from the model (i.e., only the virtual links with compromised capacity need to be considered);

- set $F_{e^{V}} \in \mathbb{R}$ within $[0,1]$ expresses the fraction of the capacity of virtual link $e^{V}$ that was compromised by a disruption event; and

- sets $P_{e^{V}}$ and $P_{p, e^{S}, e^{V}} \in \mathbb{B}$ preserve the previous mapping of the active paths of virtual link $e^{V} ; P_{e^{V}}$ is used to enumerate the paths, while $P_{p, e^{S}, e^{V}}$ indicates if the active path $p$ of virtual link $e^{V}$ contains substrate link $e^{S}$.

Objective. maximize the residual bandwidth recovered after a disruption event. Therefore, the function

$$
\max T=\sum_{\forall e^{V} \in E^{V}} \sum_{\forall p \in P_{e} V} Q_{p, e^{V}}
$$

attempts to allocate all residual bandwidth over the set of active paths. In order to avoid waisting resources, this model is subject to two constraints. The first constraint (6) guarantees that the amount of bandwidth reallocated cannot be greater than the amount compromised by an attack. The second constraint (7) ensures that the allocated bandwidth remains within the residual capacity of the substrate links.

\section{Constraints.}

- The sum of all instances of variables $Q_{p, e^{V}}$ of a given virtual link $e^{V}$ needs to be upper bounded by $F_{e^{V}}$; consequently, the total amount of additional bandwidth requested by this virtual link (i.e., $\sum_{\forall p \in P_{e} V} Q_{p, e^{V}}$ ) will not surpass $(\leq)$ the amount it actually needs $\left(F_{e^{V}}\right)$ :

$$
\sum_{\forall p \in P_{e} V} Q_{p, e^{V}} \leq F_{e^{V}}, \quad \forall e^{V} \in \dot{E}^{V}
$$

- If a substrate link $e^{S}$ is used to embed any active path of any virtual link (i.e., when $P_{p, e^{S}, e^{V}}=1$ ), then the sum of all multiplications of variable $Q_{p, e^{V}}$ by value $b\left(e^{V}\right)$ is upper bounded by $b\left(e^{S}\right)$; accordingly, the total amount of additional bandwidth requested by all compromised virtual links $\left[\sum_{\forall e^{V} \in E^{V}} \sum_{\forall p \in P_{e} V} P_{p, e^{S}, e^{V}} \cdot Q_{p, e^{V}} \cdot b\left(e^{V}\right)\right]$ from a single substrate link $e^{S}$ 
will not exceed $(\leq)$ the substrate capacity of this link $\left[b\left(e^{S}\right)\right]$ :

$$
\sum_{\forall e^{V} \in E^{V}} \sum_{\forall p \in P_{e^{V}}} P_{p, e^{S}, e^{V}} \cdot Q_{p, e^{V}} \cdot b\left(e^{V}\right) \leq b\left(e^{S}\right), \quad \forall e^{S} \in \hat{E}^{S} .
$$

\section{Evaluation}

We conducted a series of experiments to measure the performance of the proposed ORE approach. Experiments were performed in a simulated environment and executed on an Intel i7 (2nd Generation) with 8 cores of $2.93 \mathrm{GHz}$ and $8 \mathrm{~GB}$ of RAM. The goal was to evaluate ORE's resilience to disruptions, lower cost in terms of resource allocation, and compute time. We developed a simulator as there is no public domain simulator that can incorporate our approach. First, we describe the simulator, followed by a validation of the simulator through a set of basic scenarios. We then present a comparison of ORE to the back-up strategy SVNE (Survivable Virtual Network Embedding) [5]. Finally, we present an extended analysis to evaluate a series of characteristics of the ORE approach in a larger scale.

\subsection{Simulator}

The simulator is composed of a main loop performing calls to the algorithms presented in the previous section. Most of the code was written in the $\mathrm{C} / \mathrm{C}++$ language, with the exception that the LP model was generated using A Mathematical Programming Language (AMPL) ${ }^{3}$. The main loop of the simulator is described in Algorithm 4.

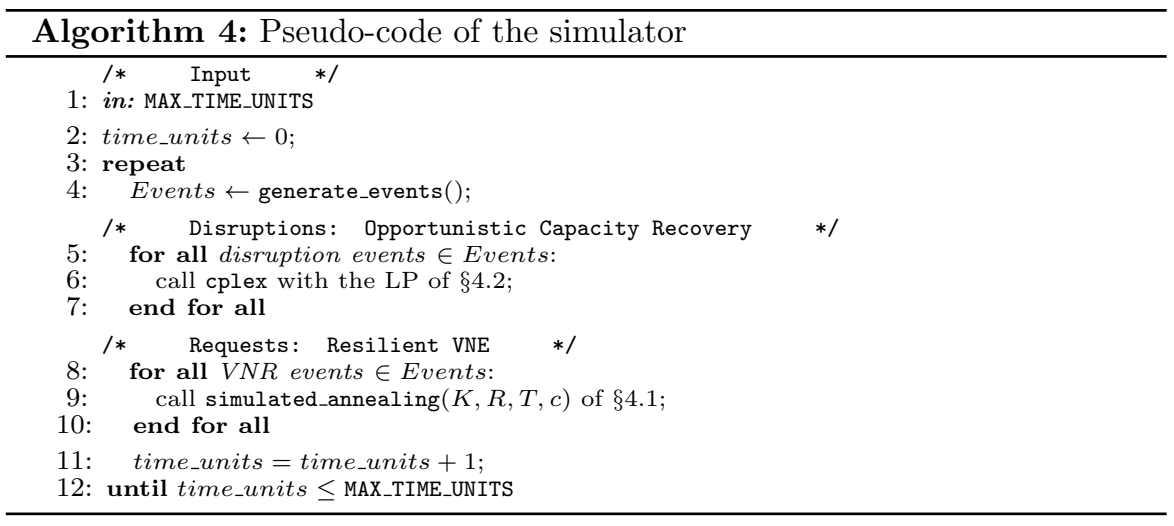

Two types of events are generated during the course of the simulation: $i$ ) disruptions and $i$ ) virtual network requests (VRNs). The former generates

\footnotetext{
${ }^{3}$ http: //www.ampl.com
} 
a call to the opportunistic capacity recovery algorithm, which is implemented as the LP model. To solve the LP, the simulator makes external calls to the CPLEX 12.3 Academic Edition ${ }^{4}$ solver. The latter generates a call to the resilient virtual network embedding algorithm, which is based on our Simulated Annealing-based allocation algorithm. Concurrent events are processed in this same order, i.e., disruption events are processed before new requests.

The events in the simulator are organized in time units where each time unit can receive one or more virtual network requests or disruptions. The time unit only finishes after concluding all steps of the allocation and/or recovery algorithms. Therefore, each time unit in a simulation may have a different duration (in seconds). More specifically, a simulation time unit may terminate instantly if there are no incoming events; it can take a few milliseconds during the execution of the LP model; or several seconds during the execution of the $\mathrm{SA}$ algorithm (the execution time is evaluated in Section 5.4).

All simulations generate scenarios where VNRs that arrive are either accepted or rejected. When plotting the acceptance rate as a function of the simulation time units, the curve exhibits a characteristic pattern ${ }^{5}$ as shown in Figure 5. First, there is a warm-up period $(0 \sim 500)$ since the substrate has sufficient resources to embed all of the arriving virtual network requests when none of them are rejected. As time passes, the system enters a stabilization phase $(500 \sim 2500)$; this happens when resources start becoming scarce and the allocation starts rejecting some requests. Finally, the allocation process stabilizes $(2500 \sim \infty)$ when the amount of resources being freed is approximately the amount demanded by new requests, and the acceptance rate converges, with a small variance, to a value $(\approx 50 \%$ in this example).

\subsection{Simulator Validation and Initial Analysis}

We conducted a series of experiments to validate the simulator through a number of configurations. These experiments are a subset of a full factorial analysis performed over the factors that affect the system. In particular, this validation considered four factors: (i) the type of topology, (ii) the type of request, (iii) the amount of resource capacity, and (iv) the use of multipath embedding Table 2 shows the list of factors and their variations. All results are reported for the "stable system" state (see the explanation for Fig. 5) and each experiment varies by only one factor.

\subsubsection{Preliminaries}

We use three types of topologies for the substrate network: ring, grid, and Barabási-Albert (BA2) [29]. The BA2 model corresponds to network topologies that grow through preferential attachment. There are two types of requests: homogeneous and heterogeneous. In homogeneous requests, all configurations of a virtual network are exactly the same, but only replicated over time. For

\footnotetext{
${ }^{4}$ http://www-01.ibm.com/software/commerce/optimization/cplex-optimizer/

${ }^{5}$ This pattern has been known in the literature $[26,27,28,17,13,12,16]$
} 


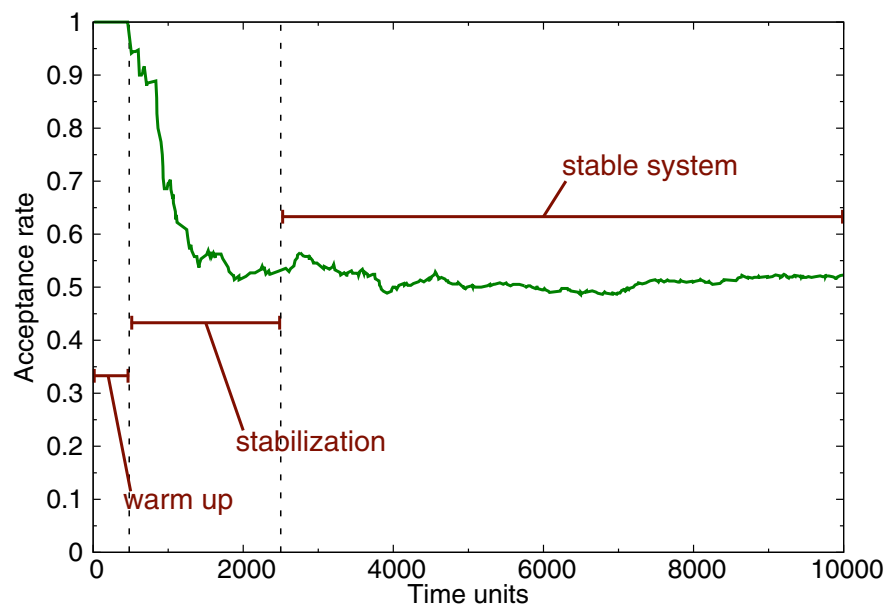

Figure 5: Illustration of a single simulation run.

Table 2: Factors considered in the simulator validation analysis.

\begin{tabular}{|c|c|}
\hline Factor & Variations \\
\hline \hline Topology & $\{$ Ring, Grid, BA2 $\}$ \\
\hline Type of Requests & $\{$ Homogeneous, Heterogeneous $\}$ \\
\hline Resource Capacity & Range $\{$ Low...High $\}$ \\
\hline Multipath Embedding & $\{$ Disabled, Enabled $\}$ \\
\hline Resilience Strategy & $\{$ ORE, SVNE[5]\} \\
\hline
\end{tabular}

heterogeneous requests, resources vary uniformly over a mean, and within a specified range. The resource capacity of the substrate network varies from low (that is, high request rejection) to high (that is, high request acceptance). The value for low network capacity is the minimum to accept at least one request, while the value for high network capacity is sufficient for near $100 \%$ acceptance. Multipath Embedding is either disabled or enabled. When disabled, virtual links are mapped on a path, while when enabled, virtual links are mapped on two paths.

All virtual network requests are represented by a simple topology consisting of 2 nodes connected by a logical link. The reason for considering this simple virtual network for validation and initial analysis is that this corresponds to an on-demand homogeneous call on a circuit-switched network. For this simplified VN request, we can then consider Erlang load given by the product of the mean number of requests per time unit and the mean duration of requests. Further, we can use the Erlang-B blocking formula [30] to approximate the theoretically maximum acceptance rate, as acceptance $=1-\operatorname{ErlangB}(\mathrm{Cap}, \mathrm{Dem})$, where ErlangB is the Erlang-B blocking probability, Cap is the aggregate resource capacity of the substrate network and and Dem is the Erlang demand this is computed as the Erlang load multiplied by the average bandwidth of VN requests. 
For this study, all substrate networks have a homogeneous capacity on links and an infinite capacity on nodes. Second, the capacity of the virtual links is either set to 2 (homogeneous case) or generated uniformly within the $[1,3]$ range with an average capacity of 2 (heterogeneous case). Third, the rate of incoming VN requests is modeled as a Poisson process with mean 2.5 requests per time unit and the duration is modeled with an exponential distribution with an average of 20 time units; thus, the total Erlang demand is 100.

Some of the characteristics in this set of validation and experiments were fixed in order to reduce the number of experiments and to facilitate validation. In particular, some simplifications were adopted in order to allow the calculation of a theoretical value for the maximum acceptance rate of the system. A more complex scenario will be considered in Section 5.4.

\subsubsection{Results}

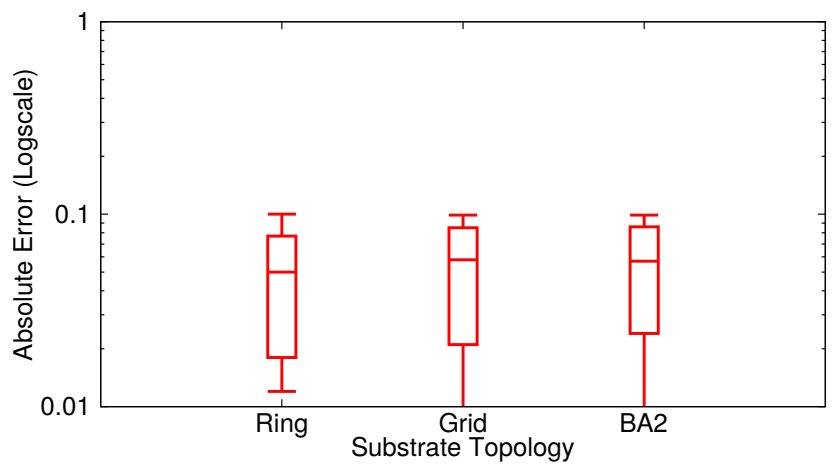

(a) Values for maximum, minimum, median, and 25\%/75\% quartiles.

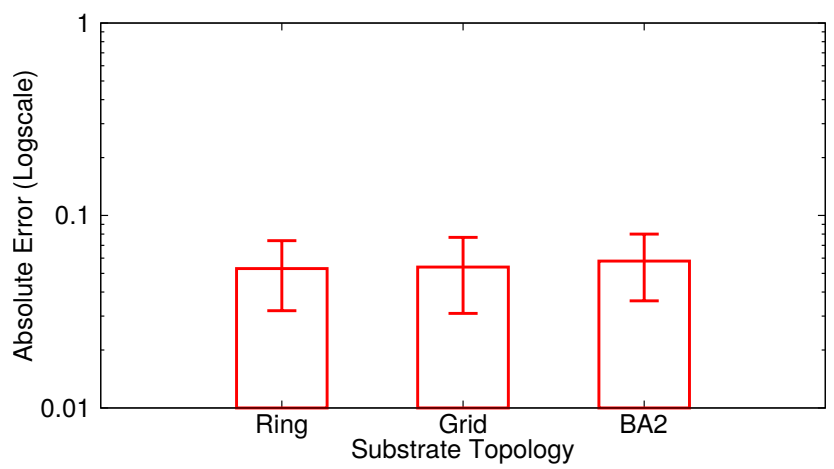

(b) Mean and confidence intervals for a $95 \%$ confidence level.

Figure 6: Topologies comparison: error between theoretical and experimental values for the Ring, Grid, and BA2 topologies. 
Figure 6 expresses the absolute error in the acceptance rate to the theoretical value (err $[x]=$ Theoretical_Acceptance_Rate $[x]$ - Experimental_Acceptance_Rate $[x]$ ) when virtual network requests were set to be heterogeneous. Topologies are configured to have the same number of links as follows: (i) the Ring was set to have 12 nodes and links; (ii) the Grid was set to have 9 nodes and 12 links (a $3 \times 3$ grid); and (iii) the BA2 was set to have 8 nodes and 12 links. Additionally, nine variations of substrate network capacity are considered, from 2 (low capacity) to 10 (high capacity). As observed in both plots, the difference in topology does not significantly influence the acceptance rate as values are similar for all scenarios.

Figure 6(a) shows the absolute error for all topologies to be in the range $[0.01,0.1]$. Moreover, the values of quartiles and medians are similar. Further, as depicted in Figure 6(b), all topologies have similar mean values, that is, around 0.05 with a $95 \%$ confidence interval of $[0.03,0.08]$.

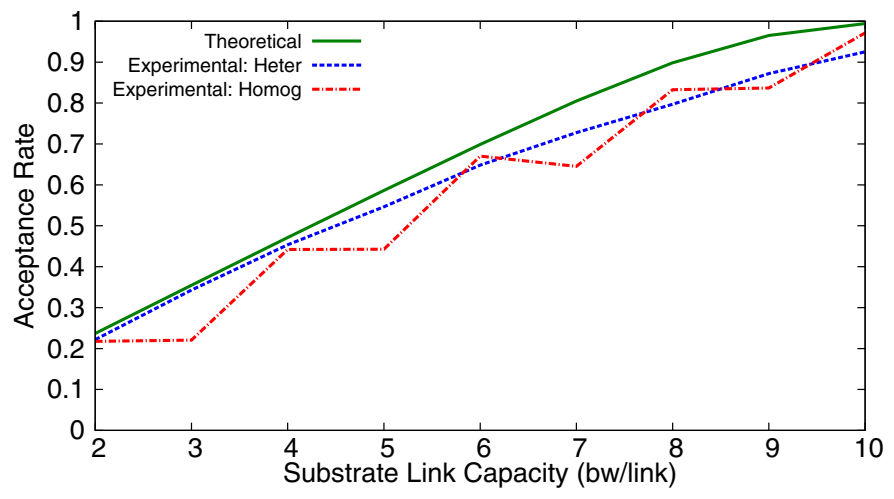

Figure 7: Homogeneity vs Heterogeneity: acceptance rate when virtual link capacity is expressed as a single value (homogeneous); or a range of values spread over a given mean (heterogeneous). In this plot, higher values are better.

Figure 7 presents a comparison of the acceptance rate (Y-Axis) for different types of requests (i.e., homogeneous or heterogeneous) over some scenarios for substrate capacity (X-Axis). In this case, the substrate topology is configured to be a Ring with 12 nodes and links (similar values were found in the other topologies, as discussed in the previous evaluation). As can be observed, the homogeneous scenario presents a step-like curve while the heterogeneous scenario is more continuous. In the homogeneous scenario, the step-like curve indicates that the allocation only increases after 2 increments in the substrate network capacity. This is because the capacity on virtual links is always set to 2 and thus requests do not fit the additional 1 unit of capacity when available. In the heterogeneous scenario, the continuous curve indicates that some requests with 1 unit of capacity are being allocated in the additional unit of link capacity. Moreover, some requests with 3 units of capacity are preferred over some with 1 and 2 units of capacity. When considering the heterogeneous curve, or 
even values for the homogeneous curve, the error to the theoretical value is less than $10 \%$ in the worst case. Moreover, both curves appear to present a growing tendency similar to the theoretical one.

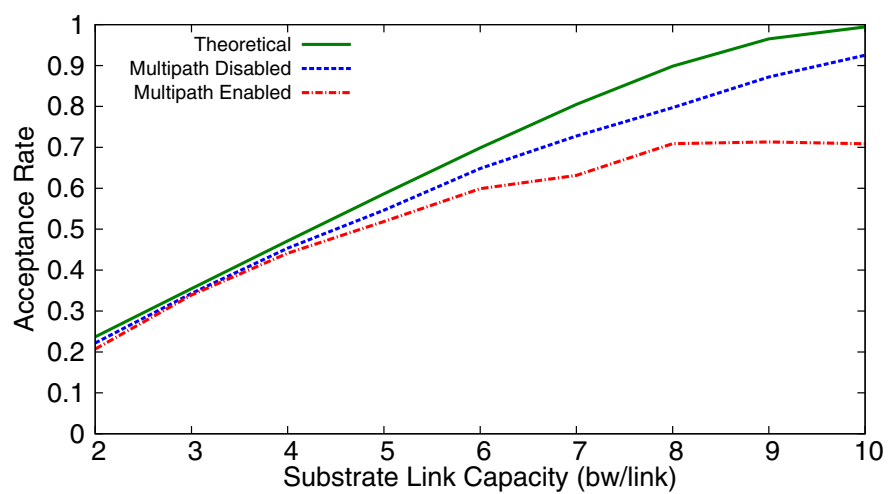

Figure 8: Multipath Embedding: effect of embedding virtual links in multiple substrate paths; study performed using a ring topology with 12 nodes. In this plot, higher values are better.

Figure 8 shows the behavior of using either single path or multipath embedding. As observed, using multiple paths decreases the acceptance rate. This happens because it is often the case that multiple paths use more resources than single paths. Accordingly, multipath embedding tends to use more substrate links in comparison to single paths.

This phenomenon is better observed in a Ring topology. For example, suppose that a virtual link is being embedded using a single substrate link. This is often the case since virtual nodes can be embedded into neighbors and, thus, the shortest path between them has a single link. In contrast, to use multipath embedding in a Ring topology, one must use the whole circumference of the Ring to connect any two nodes. This is because the first path may use a single substrate link, but the second would need to go around the whole ring using the other links. A deeper evaluation of this behaviour can be found at [31].

\subsection{Comparison of ORE to a Backup-based Strategy}

ORE provides resilience without the use of backup resources. This means that resilience is offered opportunistically (i.e., whenever there is sufficient residual capacity). To measure the efficiency of opportunistic resilience, we also performed a comparison with a backup-based strategy. First we highlighted the basics for this comparison.

Backup-based strategy for comparison. Among possible choices, we implemented SVNE (Survivable Virtual Network Embedding) [5] as it generalizes the main concept of backup-based strategies. SVNE is a link protection strategy. It protects the capacity of a single substrate link by redirecting traffic through substrate paths. SVNE is composed of two phases, offline and online. 
During the offline phase, SVNE reserves a fraction of the capacity of every substrate link as backup (say 10\%). Then, it pre-calculates paths for every substrate link. Then, in the online phase, SVNE redirects the capacity embedded in a disrupted substrate link through the backup resources of the pre-calculated paths.

Disruption model. Events are generated during the execution of the simulation. These events are modeled by a Poisson process with a mean of 0.01 per time unit and a mean duration of 1 time unit. Moreover, SVNE is designed to sustain substrate link failures only. Therefore, each disruption is assumed to be the worst case in this scenario; i.e., it disables the substrate link with the highest utilization.

Parametrization. Both ORE and SVNE require parametrization to perform properly against disruptions. Moreover, the parameters are of different natures. In particular, ORE requires the amount of paths per virtual link; the amount of bandwidth loss decreases as the number of paths increases. In contrast, SVNE needs the percentage of resources reserved as backups; the amount of bandwidth loss decreases as the percentage increases. The common factor among both strategies is the amount of bandwidth loss (or bandwidth protected). Therefore, to provide a fair comparison, we evaluated both strategies and measured bandwidth loss provided by each scheme under a range of settings.

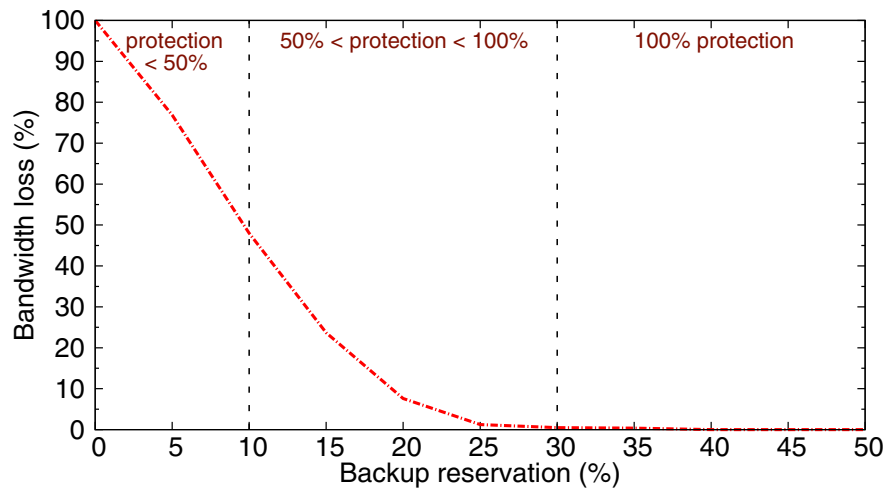

Figure 9: $S V N E$ : bandwidth loss after single link disruptions; study performed using a BA2 topology with 12 nodes and 38 links. Backup reservation varies from $0 \%$ to $50 \%$ in $5 \%$ increments. In this plot, lower values are better.

The first configuration that is equivalent to both strategies is a lower bound parameter that disables protection. In ORE, this happens by setting the number of paths to 1, while in SVNE this happens by setting the resource reservation to $0 \%$. The next set of configurations are defined by increasing the parameters of both strategies and identifying similarities in the bandwidth protection.

Figure 9 shows the performance of SVNE regarding bandwidth loss. In this experiment, backup reservation varies from $0 \%$ to $50 \%$ in $5 \%$ increments. Results are organized in three intervals, according to the amount of protected 
Table 3: Configurations of resilience strategies.

\begin{tabular}{|c|c|c|c|}
\hline Configuration & $\begin{array}{c}\text { Average protection } \\
\text { against bandwidth loss }\end{array}$ & $\begin{array}{c}\text { ORE } \\
\text { (paths/virtual links) }\end{array}$ & $\begin{array}{c}\text { SVNE[5] } \\
\text { (backup reservation) }\end{array}$ \\
\hline \hline C1 & $0 \%$ & 1 & $0 \%$ \\
\hline C2 & $>50 \%$ & 2 & $10 \%$ \\
\hline C3 & $>75 \%$ & 4 & $15 \%$ \\
\hline C4 & $>90 \%$ & 6 & $20 \%$ \\
\hline C5 & $>95 \%$ & 8 & $25 \%$ \\
\hline C6 & $>97.5 \%$ & 10 & $30 \%$ \\
\hline
\end{tabular}

bandwidth. The first represents configurations that provide less than $50 \%$ protection. The second represents configurations that provide at least $50 \%$ protection and can offer nearly $100 \%$ protection. Finally, the third interval represents all configurations that provide $100 \%$ protection. We choose the second interval $[10 \%, 30 \%]$ because it provides better trade-offs between bandwidth protection and resource reservation. In short, bandwidth protection is too low in the first and resource reservation is too high in the third. Similar values are found in SVNE by configuring the number of paths from 2 to 10 in increments of 2 . Table 3 summarizes the six configurations selected for comparing each strategy.

Figure 10 shows the behavior of ORE and SVNE in terms of bandwidth loss. As observed, ORE can offer protection comparable to SVNE with configurations $\mathrm{C} 1$ and $\mathrm{C} 2$, but SVNE outperforms ORE from C3 onwards. When recovery is active, ORE performs better than SVNE in configurations $\mathrm{C} 2$ and $\mathrm{C} 3$, and worse in configurations $\mathrm{C} 4, \mathrm{C} 5$, and $\mathrm{C} 6$. This behavior is explained as follows. On the one hand, ORE outperforms SVNE when the amount of residual resources in the substrate is greater than the amount reserved as backup. In particular, in C2 and C3, ORE is not limited by $10 \%$ or $15 \%$ of the substrate resources - ORE can use as much bandwidth as there was available when the disruptions occurred. On the other hand, SVNE outperforms ORE when it has more resources reserved as backup than the amount of residual resources in the substrate. Specifically, in C4, C5, and C6, resource reservations are greater than the residual bandwidth when the disruptions occurred.

Figure 11 presents the behavior of both strategies in terms of the acceptance rate. In this set of experiments, ORE performs better than SVNE for configurations $\mathrm{C} 2$ to $\mathrm{C} 6$. This happens because the acceptance rate is affected by resource reservation. SVNE outperforms ORE if configured with less than a $10 \%$ the backup reservation. However, in this case, the bandwidth loss would be greater than $75 \%$ (as shown in Fig. 9).

Some considerations are noteworthy. First, if the capacity is very high or the demand is very low, SVNE can theoretically outperform ORE in both acceptance rate and bandwidth loss. However, this requires extreme scenarios and careful parametrization. For example, if the substrate network can accept all requests at half of the capacity, then the backup reservation can be as high as $50 \%$. This ensures $100 \%$ protection, but half of the resources are wasted when no disruption occurs. Second, although ORE can potentially achieve $100 \%$ protection, it cannot make any guarantees since the capacity used for recovery is 


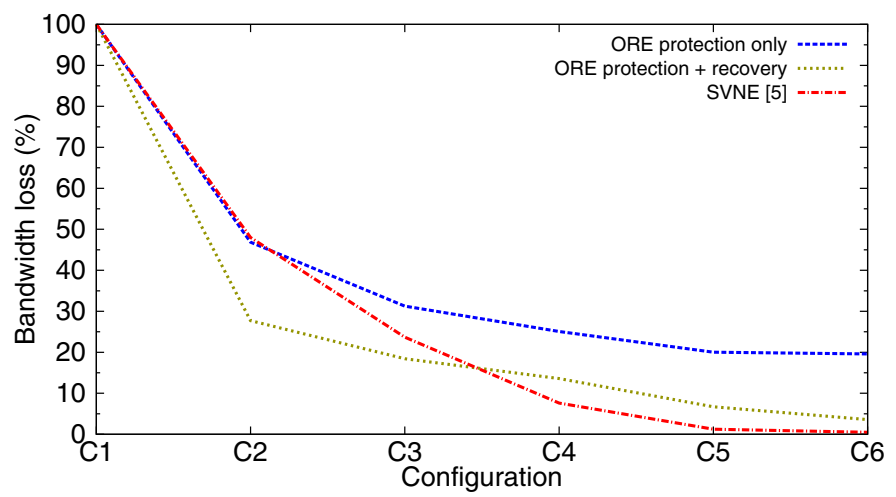

Figure 10: Resilience Strategy: bandwidth loss after single link disruptions for both ORE and SVNE; study performed using a BA2 topology with 12 nodes and 38 links. Algorithms use 6 configurations, as described in Table 3 . In this plot, lower values are better.

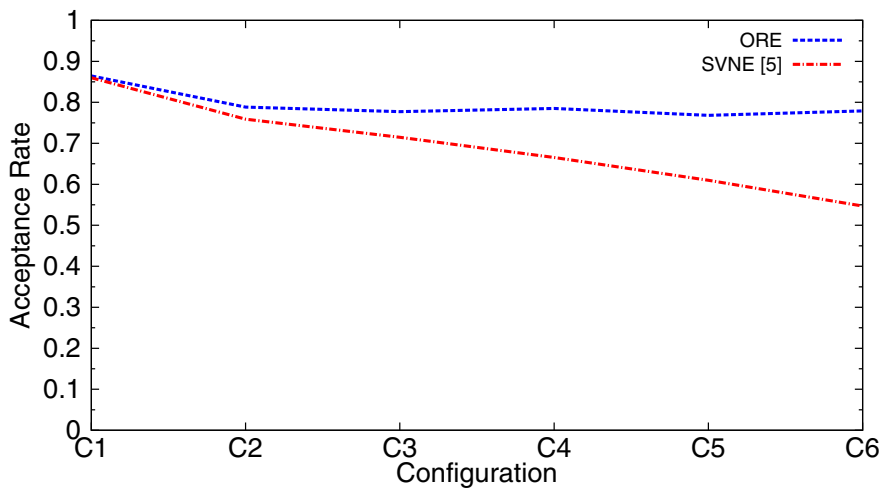

Figure 11: Resilience Strategy: acceptance rate in for both ORE and SVNE; study performed using a BA2 topology with 12 nodes and 38 links. Algorithms use 6 configurations, as described in Table 3. In this plot, higher values are better.

the same as the one used for allocations (i.e., resilience is opportunistic). SVNE, in contrast, can guarantee $100 \%$ protection if half the capacity is set as backup. Finally, the performance is sensitive to the network topology. As will be demonstrated in Section 5.4, path diversity plays a key role in recoverability. For a related work on backup strategies along with restoration in an embedded environment for circuit-switched networks, the reader is referred to an earlier work [32].

\subsection{Extended Analysis}

As described earlier in the paper, ORE has two main roles: i) to prevent against the impact of disruptions by efficiently allocating the virtual links into 
multiple paths; and $i$ ) to react against these disruptions, whenever possible, by reallocating compromised capacity over a set of active paths. This section extends the previous evaluation by $(a)$ considering a bigger topology, $(b)$ evaluating other metrics, and $(c)$ quantifying the time it takes for allocating virtual network requests and reacting against disruptions.

Similar to the previous subsection, all samples were collected after the allocation process stabilized (see the explanation of Figure 5). Moreover, unless otherwise specified, error bars represent intervals for a $95 \%$ confidence level.

\subsubsection{Settings}

Workload. The workload is composed of one substrate network, several virtual networks, and arrival and departure rates for VNR and disruption events. Each network is composed of nodes and links with cpu and bandwidth requirements, respectively. Event arrivals and departures follow known distributions. The choice of parameters presented next was based on related work $[16,13]$.

Both the substrate network topology and the virtual network topologies were created using BRITE $^{6}$ with the Barabási-Albert (BA2) [29] model. The substrate network is composed of 50 nodes and 194 links. The CPU of the nodes and the bandwidth of the links are uniformly distributed in $[50,100]$. Virtual networks vary in size; the number of nodes in a virtual network topology is uniformly distributed between 2 and 10, while the number of links is equal to the number of nodes minus one $\left(\left|N^{V}\right|-1\right)$. The capacity of virtual nodes and virtual links is uniformly distributed within $[5,15]$ and $[15,30]$, respectively. Similar to previous work, units are kept abstract; they could represent Mbps on links or time slices on nodes.

There are two types of events in this environment: virtual network requests (VNRs), and disruptions. The load of VRNs is of 70 Erlangs. More precisely, the arrival rate is modeled by a Poisson process with an average of 0.7 per time unit, whereas duration is modeled by an exponential distribution with an average of 100 time units.

The load of disruptions is of 0.05 Erlangs, which is modeled by a Poisson process with a mean of 0.01 per time unit and duration of 5 time units. Each disruption follows the worst case scenario and removes the substrate node that has the greatest bandwidth allocation on its links; this is a common assumption made to model strategic attacks [33]. Each simulation was executed during 10000 time units. This choice was made because, at this point, all simulations had already stabilized. The results were collected in the stable system state (see the explanation for Figure 5). During this state, each experiment generated approximately 7000 requests and 100 disruptions.

Tunning SA parameters. As presented previously (Section 4.1), the parameters of SA are as follows: the maximum number of outer steps, the maximum number of inner steps (also meaning the number of neighbors per outer step), the temperature, and the cooling factor. These parameters are denoted by

\footnotetext{
${ }^{6}$ http://www.cs.bu.edu/brite
} 
$K, R, T$, and $c$, respectively. The main idea behind them is that by increasing the number of outer $(K)$ and inner $(R)$ steps, and adjusting the temperature $(T)$ and cooling factor $(c)$, the SA algorithm can achieve better solutions. However, better solutions come at the cost of more computation. Specifically, notice that $K$ and $R$ have multiplicative costs, that is, increasing $K$ by 1 results in $R$ additional substeps. Therefore, the SA parameters should be set to an appropriate value.

In this work, they were first empirically defined after an initial study, and then tuned until there was no sensitive improvement in the quality of the solutions. The final set of parameters used in the rest of the experiments were the following: the number of outer steps $(K)$ was set to 80 ; the number of neighbors per step $(R)$ to 2000 ; the temperature $(T)$ to 3000 ; and the cooling factor $(c)$ to 0.96 . This combination of parameters required 160,000 (160 thousand) steps to reach an acceptance rate of approximately $72 \%$.

\subsubsection{Results}

A) ORE mitigates the impact of the substrate node disruptions

The previous analysis (depicted in Fig. 10) studied disruptions in substrate links only. Figure 12(a) shows how ORE protects virtual links from bandwidth loss in substrate node disruptions. The plot depicts the average bandwidth loss ( $y$ axis) as a function of the number of paths to be used when embedding a virtual link ( $x$ axis); hence, the lower the value, the better it is. The baseline has only one path per virtual link. In this case, when a disruption occurs, ORE cannot protect any bandwidth. This causes the virtual links to become inoperable. Intuitively, increasing the number of paths should make the network more resilient.

As expected, the effectiveness of ORE's protection strategy is proportional to the number of paths per virtual link. Observe that by using 2 paths per virtual link, ORE decreases bandwidth loss to approximately $61 \%$; that is, it protects about $39 \%$. Further, by increasing the number of paths to 4 and 6 , ORE can protect $65 \%$ and $79 \%$ of the bandwidth, respectively. The potential for bandwidth protection stabilizes around $85 \%$, when using 8 and 10 paths per virtual link.

Figure 12(b) shows the relative gain in bandwidth protection achieved by ORE. The $y$ axis represents protected bandwidth, while the $x$ axis represents the number of paths per virtual link. In other words, the plot depicts how much extra bandwidth was saved by each increment in the number of paths. Although the efficiency of ORE's strategies increases with the number of paths, this behavior is nonlinear. When using 2 and 4 paths, ORE protects $39 \%$ and $65 \%$ of the bandwidth, respectively. This means a gain of approximately $26 \%$ in bandwidth protection. In comparison, this gain is reduced to about $14 \%$ when the number of paths increases to 6 . Moreover, the relative gain of increasing from 6 to 8 paths is only $4 \%$ and from 8 to 10 is negligible (around 1\%). This happens because the substrate network has a limited amount of disjoint paths available to embed each virtual link. The next experiment provides further evidence of this behavior. 


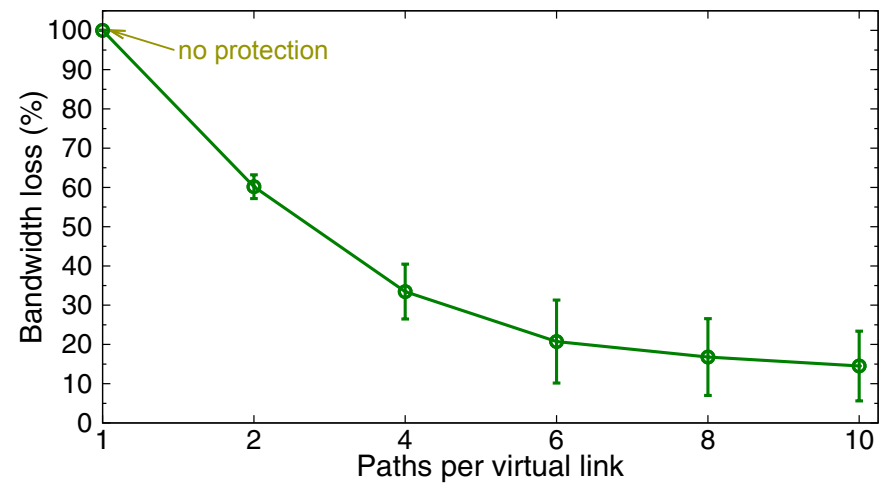

(a) Bandwidth loss on scenarios with 1 to 10 paths per virtual link.

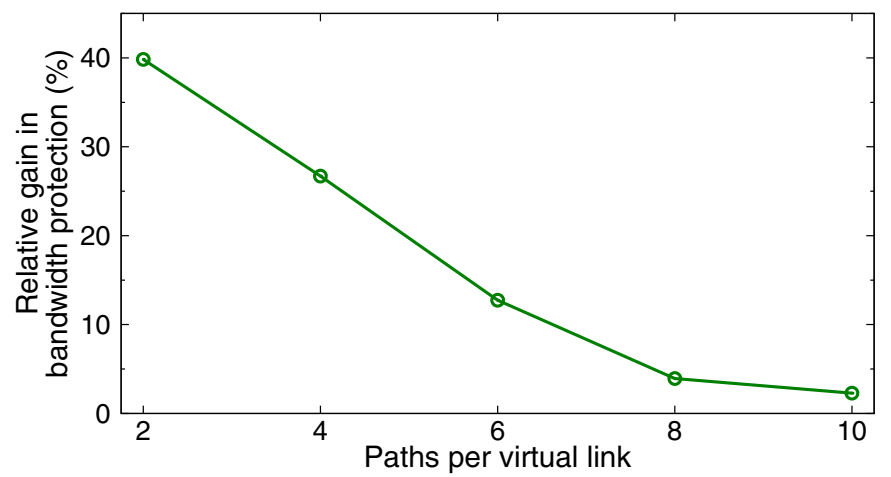

(b) Relative gain in bandwidth protection.

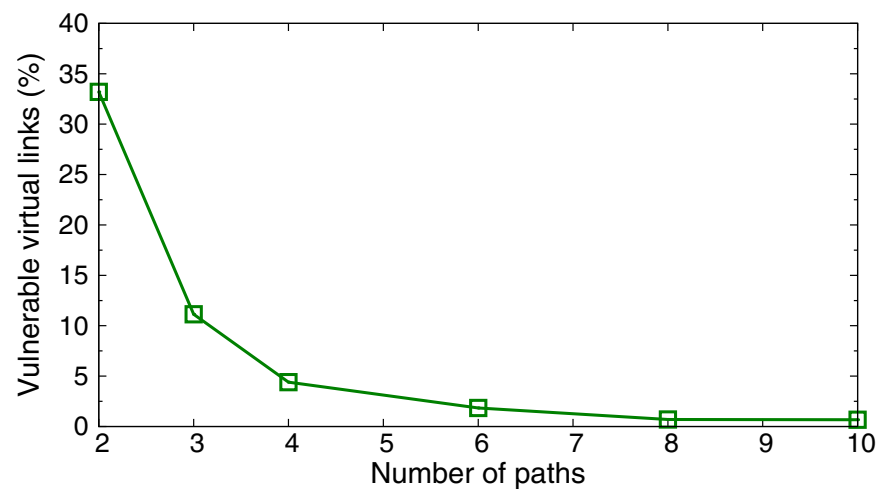

(c) Percentage of virtual links vulnerable to attacks on critical links in a substrate network with 50 nodes and 194 links.

Figure 12: Analysis of the gain in bandwidth protection and recoverability according to the number of paths used to embed each virtual link. 
B) Multipath allocation reduces the severity of disruptions in SRLGs

As previously discussed, shared risk link group (SRLG) is defined as a substrate link that concentrates the full capacity of a virtual link by a circumstance of the substrate network and/or the allocation procedure. According to this definition, if an attack on such link succeeds, one or more virtual links would be fully compromised. More specifically, this situation happens when all paths of a given virtual link share, at least, one substrate link. For example, if a virtual link uses two paths, then both paths should be disjoint to be protected from this type of attack. Otherwise, a planned attack on a SRLG would cause the virtual link to become unresponsive.

Figure 12(c) shows how the embedding approach used by ORE can decrease the vulnerability to such attacks. Vulnerability is measured by seeing how many virtual links have all their paths sharing a same substrate link. Axis $x$ and $y$ respectively represent the number of paths used in the embedding process and the percentage of virtual links vulnerable to this type of attack; therefore, the lower the value, the better. As can be observed, when considering 2 paths per virtual link, approximately $34 \%$ of virtual links are vulnerable to a strategically planned attack in a critical link. By increasing the number of paths, the percentage of vulnerable virtual links decreases to $12 \%$ (using 3 paths), then $5 \%$ (using 4 paths), and finally stabilizes around 1\% 2\% (using 6 to 10 paths). Based on these results, it is possible to infer that the protection against this type of attack is proportional to the number of paths. This behavior can be further analyzed with a full view of path similarities.

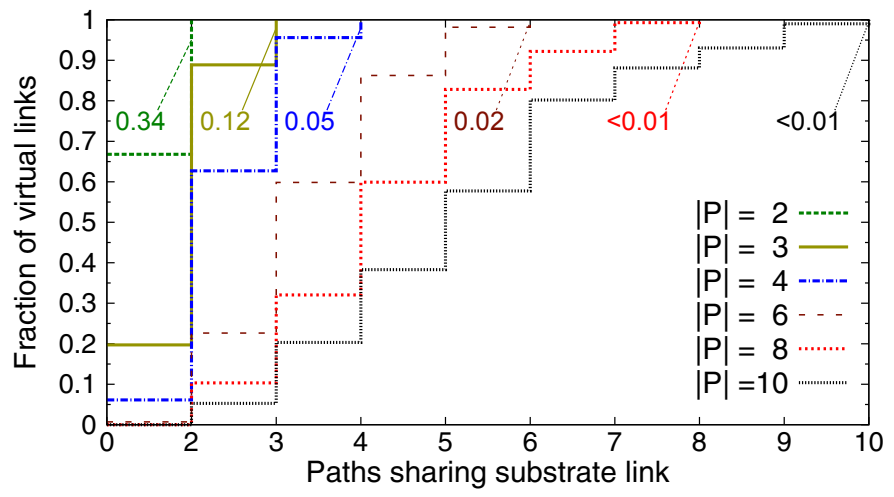

Figure 13: CDF displaying path similarity of virtual links in a substrate network with 50 nodes and 194 links.

The CDF (Cumulative Distribution Function) presented in Figure 13 quantifies the virtual links' similarity (i.e., the amount of paths that share a substrate link). While the $y$ axis represents the fraction of virtual links embedded into the substrate, the $x$ axis represents how many paths of a same virtual link are overlapping. Numbers indicate the amount of virtual links vulnerable to attacks in critical links (as recently discussed). The special case " 0 " is the equivalent to 
a disjoint embedding, that is, the paths selected to embed a given link do not share any substrate link. Notice that, as the number of paths increases, the likelihood of all of them being embedded on the same substrate link decreases. This characteristic is desirable because it increases the amount of substrate resources an attacker has to compromise to disconnect a virtual network.

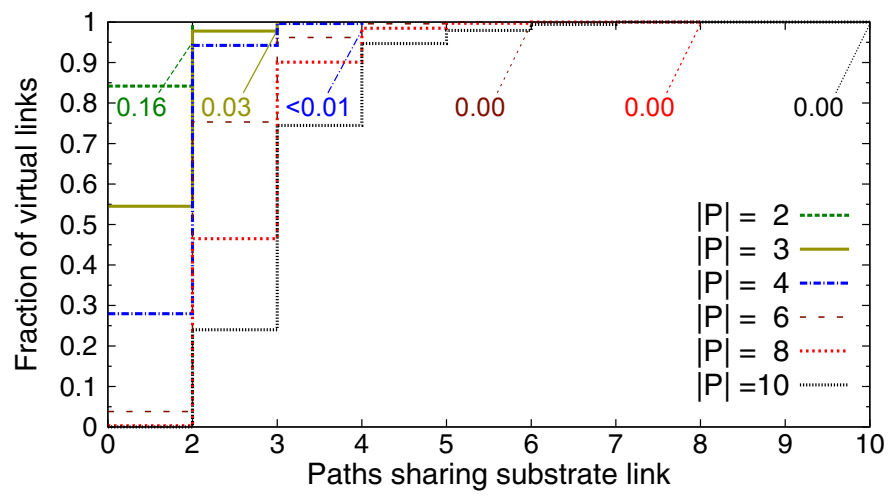

Figure 14: CDF displaying path similarity of virtual links in a substrate network with 50 nodes and 380 links.

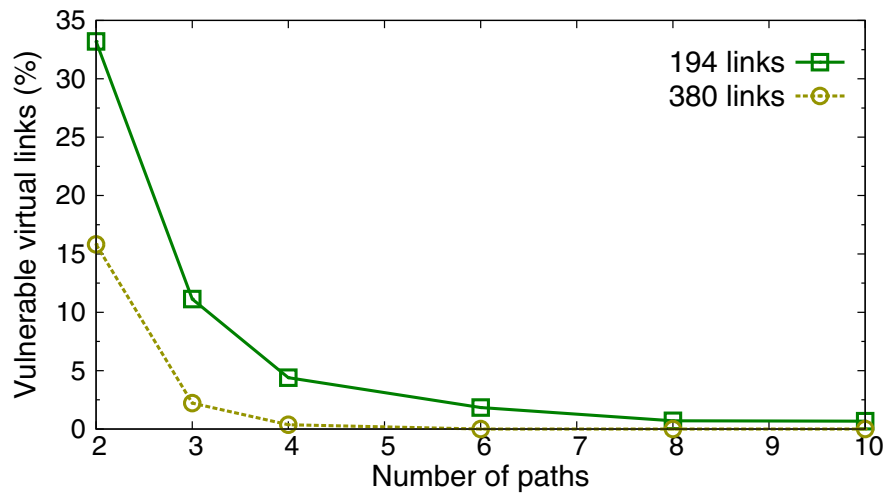

Figure 15: Comparison of the percentage of virtual links vulnerable to attacks on critical links, in scenarios with 194 and 380 substrate links.

Further analyzing Figure 13, it is possible to see that the fraction of virtual links with disjoint paths also decreases. The importance of disjoint paths is that if one or more paths share the same underling substrate link, the efficiency of the opportunistic recovery strategy is limited by the capacity of the shared link. When virtual links are embedded on 2 paths, $66 \%$ of these virtual links have disjoint paths (i.e., do not share any substrate link). This decreases to $20 \%$ when the number of paths per virtual link increases to 3 , and then $6 \%$ with 
4 paths per virtual link. Further, this value is reduced to less than $1 \%$ for a higher number of paths. A possible explanation for this behavior is that the amount of path diversity available in the substrate network hinders the ability of the algorithm to avoid similarity. This inference is evaluated by increasing the number of substrate links without varying the number of nodes, thus enhancing path diversity.

Figure 14 shows a CDF similar to the previous one for a substrate network of 50 nodes and 380 links. The number of nodes remained the same as in Figure 13, but the number of links almost doubled. According to the plot, the previous supposition sustains and ORE is able to reduce path similarity (i.e., the CDF is shifted towards the left). As can be observed, the fraction of virtual links embedded in disjoint paths is higher than the previous scenario; for example, with two paths per virtual link, this number increases from $0.66(66 \%)$ to 0.84 (84\%). Similarly, the fraction of virtual links in which all paths share a single substrate link is reduced. Consequently, as shown in Figure 15, the percentage of virtual links vulnerable to attacks in critical links is reduced significantly, reaching less than 1\% (approximately $0.5 \%$ ) when using 4 paths per virtual link. In comparison, in the previous allocation this number was more than 10 times as high.

C) ORE's computing time is linear with respect to the number of paths per virtual link and the size of the VNR

Figures 16 and 17 present the time to compute the proactive strategy and reactive strategy, respectively (experiments using Intel i7 2nd Generation and CPLEX Academic Edition). Figure 16 depicts time as a function of (a) the size of virtual network requests; and (b) the number of paths per virtual link. Since both parameters affect the time to compute a solution, one is kept constant in each plot. The size of VNRs is represented by the number of nodes in each request. As observed, time is linearly proportional to the size of both inputs. As inputs grow in size, ORE is expected to grow linearly. Further, it is possible to see that, in the selected scenario, ORE can embed each virtual network request in under a minute.

The two strategies comprising ORE work at two different time frames. The proactive strategy takes at most half a minute to compute (Figure 16), while the reactive strategy takes less than 15 milliseconds to compute (Figure 17). This is because the strategies were designed with different goals. The proactive one is intended to provide efficient allocation and opportunistic protection (as discussed in Sections 3.1 and 4.1) at the time of the initial VN request embedding on the substrate network. The reactive strategy is aimed at a fast reaction against disruptions (Sections 3.2 and 4.2). Note that we use a relatively low powered machine (i7) for this study. In a production environment, the proactive phase is employable in real-time or near real-time since a much more powerful machine can be used to cut down the compute time to a few seconds. 


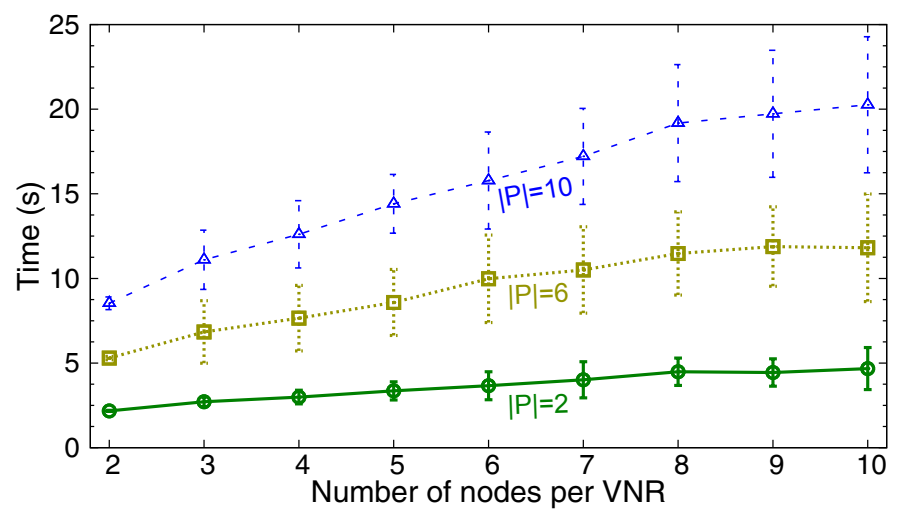

(a)

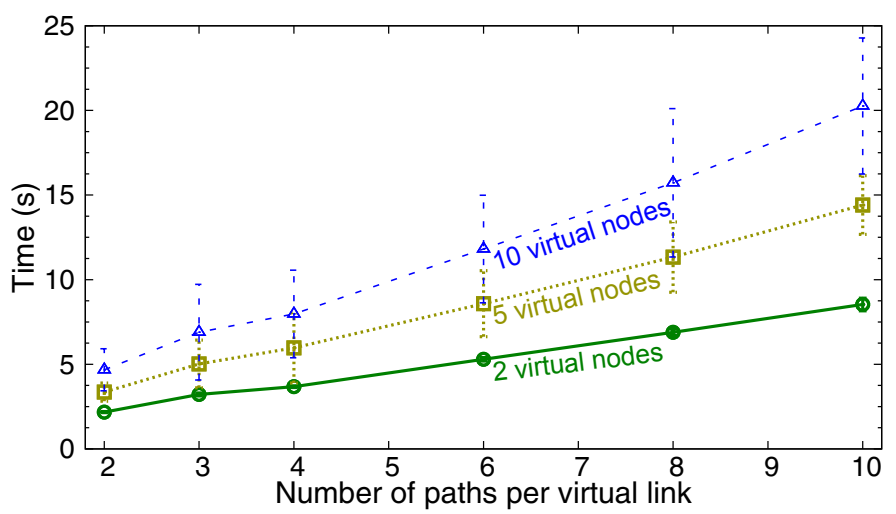

(b)

Figure 16: Mean time to compute allocation.

\section{Related Work}

This section reviews the literature related to resilient virtual network embedding. We first compare the common aspects between Resilient Virtual Network Embedding (VNE) and traditional network protection. Then, we discuss recent papers related to Resilient VNE.

\subsection{Traditional Network Protection}

Traditional network protection differs from Resilient VNE in several ways. Differences mostly concern the online/offline natures of the problems, network topology, and attributes in consideration.

As discussed in Section 2.1, VNE optimization problems can be either online or offline; that is, whether virtual network allocation requests arrive on-demand, without any prior knowledge, or wheter they can be determined beforehand. 


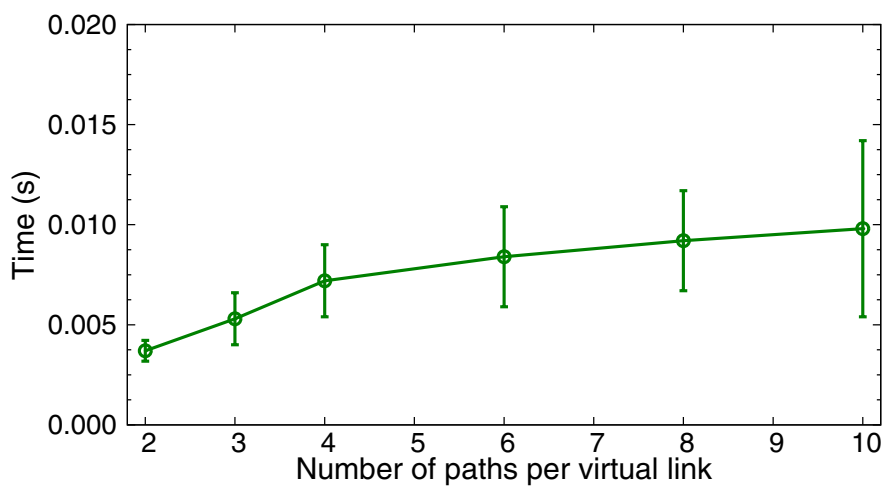

Figure 17: Mean time to compute restoration. In this plot, error bars indicate standard deviation, since the interval for a $95 \%$ confidence level was too small.

Similarly, traditional network optimization problems can also be offline or online, depending on the focus of the models. The offline version is commonly known as network protection design (or survivable network design) [34]. This subject is well studied in the context of IP-over-optical networks; for example, see [35, $36,37,38,39,23,24,25,40]$. However, most of these networks consider the embedded virtual networks to be static, while with network virtualization we anticipate dynamic virtual network embedding in real-time [3]. Hence, offline models are not suitable for VNE because requests are not known in advance, and assuming this knowledge would break the purpose of using substrate resources on-demand.

Traditional network optimization problems can also be modeled as an online problem when requests are switched or routed on a per-unit basis and are of short duration [34]. For example, on-demand optical services in a SONET/SDH network [41] are similar to on-demand virtual network requests in VNE. However, connections represent pairs of pre-defined source/sink nodes, in contrast to customized virtual network topologies. Moreover, traditional networks only consider link-related attributes, whereas virtual networks request attributes on both the nodes and the links [3]. Therefore, even when the problem is formulated online, traditional network protection proposals are unsuitable for network virtualization as node embedding (i.e., placement and requirements) is not considered.

Finally, restoration can also be performed by routing schemes. Generally, they will update the forwarding tables to account for an unresponsive link or router. In this context, the most related scheme is Valiant Load Balancing (VLB). VLB is a multipath routing scheme used in datacenter networks [42] and multi-layer optical networks [43] to achieve higher throughput and resilience. Upon disruptions, VLB identifies affected paths and sends traffic over the remaining paths. This is orthogonal to VNE since the virtual networks have their own routing algorithms, possibly containing customized restoration mechanisms. 


\subsection{Resilience in Virtual Network Embedding}

Resilience in virtual networks is generally provided by reserving additional resources as backup during embedding. The idea is intuitive: create additional copies of the original resources (denoted as primary) of a virtual network and allocate these copies in disjoint substrate resources when solving the VNE problem.

Virtual links are protected in two ways: physical link protection (through preallocation of detours) [5,6]; or physical path protection (through restoration paths) $[44,6]$. Physical link protection aims to protect each individual link, regardless of the virtual links embedded in it. The technique consists of precalculating k-alternative paths (dubbed detours) for the endpoints of each substrate link; then, when a disruption occurs, the affected virtual links are rerouted through these detours. Physical path protection is similar to protection mechanisms used in IP over MPLS networks. It consists of restoration paths - that are extra disjoint paths - for embedding each virtual link. In this case, the extra paths are calculated and allocated at the embedding phase and used when a disruption affects the primary embedding.

Virtual node protection $[7,8]$ or joint, node-link protection [45] are also studied. Broadly, strategies consist of first replicating virtual nodes on separate physical locations and then using restoration paths to protect virtual links.

The use of backup resources is effective as the additional resources are mapped disjointly in order to avoid co-related or cascading disruptions. The main disadvantage in this strategy is that the use of backup resources may become too expensive. The extra overhead in resource consumption may hinder the ability of the substrate network to accept future requests. Further, they are wasted if no disruption occurs.

This paper extends a previous proposal [46] on several aspects. First, it enhances the main allocation metaheuristic with the addition of a Local Improvement algorithm. Second, a wider set of experiments is presented. Third, the methodology employed for experimentation, the evaluation scenarios, and the workload configurations are discussed in more depth. Fourth, it is shown how ORE adapts to the topology and benefits from the available path diversity to better protect virtual networks from disruptions in the substrate network.

\section{Conclusions}

In this paper, we presented a novel virtual network embedding algorithm based on the Simulated Annealing metaheuristic for protecting virtual networks against disruptions in the physical substrate. Unlike previous work, our approach does not need to set aside backup resources. Rather, it employs multiple path virtual link embedding and opportunistic recovery to mitigate the impact of disruptions.

Results show that the proposed approach provides resilience to virtual networks with a higher long-term acceptance rate. In future work, we aim at investigating multi-layer optimization to avoid overlapping protection mechanisms. 


\section{Acknowledgment}

This work has been supported by the following grants: FP7 and CNPq Project SecFuNet (FP7-ICT-2011-EU-Brazil), RNP CTIC program (Project ReVir), PRONEM/FAPERGS/CNPq (Project NPRV), CNPq (Project Phoenix), CNPq (Project ProSEG), CNPq (Science Without Borders Program), Microsoft Azure for Research grant award, and National Science Foundation Grant No. 1217736 .

\section{References}

[1] N. M. K. Chowdhury, R. Boutaba, A survey of network virtualization, Computer Networks 54 (5) (2010) 862-876.

[2] A. Khan, A. Zugenmaier, D. Jurca, W. Kellerer, Network virtualization: a hypervisor for the internet?, IEEE Communications Magazine 50 (1) (2012) $136-143$.

[3] A. Belbekkouche, M. Hasan, A. Karmouch, Resource discovery and allocation in network virtualization, IEEE Communications Surveys \& Tutorials 14 (4) (2012) 1114-1128.

[4] M. Luizelli, L. R. Bays, L. S. Buriol, M. P. Barcellos, L. P. Gaspary, Characterizing the impact of network substrate topologies on virtual network embedding, in: Proceedings of the 9th International Conference on Network and Service Management, IFIP CNSM, Zurich, Switzerland, 2013, pp. $42-50$.

[5] M. Rahman, R. Boutaba, Svne: Survivable virtual network embedding algorithms for network virtualization, Network and Service Management, IEEE Transactions on 10 (2) (2013) 105-118.

[6] T. Guo, N. Wang, K. Moessner, R. Tafazolli, Shared backup network provision for virtual network embedding, in: Proceedings of the 2011 IEEE International Conference on Communications, IEEE ICC, Kyoto, Japan, 2011, pp. 1-5.

[7] W.-L. Yeow, C. Westphal, U. C. Kozat, Designing and embedding reliable virtual infrastructures, in: Proceedings of the 2nd SIGCOMM workshop on Virtualized Infrastructure Systems and Architectures, VISA, ACM SIGCOMM, New Delhi, India, 2010, pp. 33-40.

[8] H. Yu, V. Anand, C. Qiao, G. Sun, Cost efficient design of survivable virtual infrastructure to recover from facility node failures, in: Proceedings of the 2011 IEEE International Conference on Communications, IEEE ICC, Kyoto, Japan, 2011, pp. 1-6. 
[9] H. Yu, C. Qiao, V. Anand, X. Liu, H. Di, G. Sun, Survivable virtual infrastructure mapping in a federated computing and networking system under single regional failures, in: Proceedings of the 53th annual IEEE Global Telecommunications Conference, IEEE Globecom, Miami, FL, USA, 2010, pp. $1-6$.

[10] R. R. Oliveira, L. R. Bays, D. S. Marcon, M. C. Neves, L. S. Buriol, L. P. Gaspary, M. P. Barcellos, Dos-resilient virtual networks through multipath embedding and opportunistic recovery, in: Proc. SAC, ACM, 2013, pp. 597-602.

[11] D. G. Andersen, Theoretical approaches to node assignment, Tech. rep., unpublished manuscript. Available from: http://goo.gl/Xl1ph. Accessed 3rd May 2013. (2002).

[12] M. Chowdhury, M. R. Rahman, R. Boutaba, Virtual network embedding with coordinated node and link mapping, in: Proceedings of the 28th Annual Joint Conference of the IEEE Computer and Communications Societies, IEEE INFOCOM, Rio de Janeiro, RJ, Brazil, 2009, pp. 783-791.

[13] X. Cheng, S. Su, Z. Zhang, K. Shuang, F. Yang, Y. Luo, J. Wang, Virtual network embedding through topology awareness and optimization, Computer Networks 56 (6) (2012) 1797-1813.

[14] M. Yu, Y. Yi, J. Rexford, M. Chiang, Rethinking virtual network embedding: substrate support for path splitting and migration, ACM SIGCOMM Computer Communication Review 38 (2008) 17-29.

[15] I. Houidi, W. Louati, W. B. Ameur, D. Zeghlache, Virtual network provisioning across multiple substrate networks, Computer Networks 55 (4) (2011) 1011-1023.

[16] M. Chowdhury, M. R. Rahman, R. Boutaba, Vineyard: Virtual network embedding algorithms with coordinated node and link mapping, IEEE/ACM Transactions on Networking 20 (1) (2012) 206-219.

[17] N. F. Butt, M. Chowdhury, R. Boutaba, Topology-awareness and reoptimization mechanism for virtual network embedding, in: M. Crovella, L. Feeney, D. Rubenstein, S. Raghavan (Eds.), NETWORKING 2010, 9th International IFIP TC 6 Networking Conference, Vol. 6091 of Lecture Notes in Computer Science, Springer Berlin/Heidelberg, Berlin, Germany, 2010, pp. 27-39.

[18] J. He, J. Rexford, Toward internet-wide multipath routing, IEEE Network 22 (2) (2008) 16-21.

[19] T. Hartman, A. Hassidim, H. Kaplan, D. Raz, M. Segalov, How to split a flow?, in: Proceedings of the 31th Annual Joint Conference of the IEEE Computer and Communications Societies, IEEE INFOCOM, Orlando, FL, USA, 2012, pp. 828-836. 
[20] M. Pióro, D. Medhi, Routing, flow, and capacity design in communication and computer networks, Elsevier, 2004.

[21] B. Fortz, M. Thorup, Internet traffic engineering by optimizing ospf weights, in: Proceedings of the 19th Annual Joint Conference of the IEEE Computer and Communications Societies, Vol. 2, IEEE INFOCOM, Tel Aviv, Israel, 2000, pp. 519-528.

[22] B. Fortz, M. Thorup, Increasing internet capacity using local search, Computational Optimization and Applications 29 (2004) 13-48.

[23] B. Rajagopalan, D. Pendarakis, D. Saha, R. S. Ramamoorthy, K. Bala, Ip over optical networks: Architectural aspects, IEEE Communications Magazine 38 (9) (2000) 94-102.

[24] D. Medhi, S. Sankarappan, Impact of a transmission facility link failure on dynamic call routing circuit-switched networks under various circuit layout policies, Journal of Network and Systems Management 1 (2) (1993) 143169.

[25] D. Medhi, A unified approach to network survivability for teletraffic networks: Models, algorithms and analysis, IEEE Transactions on Communications 42 (234) (1994) 534-548.

[26] G. P. Alkmim, D. M. Batista, N. L. S. Fonseca, Optimal mapping of virtual networks, in: Proceedings of the 54th annual IEEE Global Telecommunications Conference, IEEE Globecom, Houston, TX, USA, 2011, pp. 1-6.

[27] G. P. Alkmim, D. M. Batista, N. L. S. Fonseca, Approximated algorithms for mapping virtual networks on network substrates, in: Proceedings of the 2012 IEEE International Conference on Communications, IEEE ICC, Ottawa, Canada, 2012.

[28] L. R. Bays, R. R. Oliveira, L. S. Buriol, M. P. Barcellos, L. P. Gaspary, Security-aware optimal resource allocation for virtual network embedding, in: Proceedings of the Mini-Conference of the 8th International Conference on Network and Service Management, IFIP CNSM, Las Vegas, NV, USA, 2012, pp. 378-384.

[29] R. Albert, A.-L. Barabási, Topology of evolving networks: Local events and universality, Physical Review Letters 85 (2000) 5234-5237.

[30] D. Medhi, K. Ramasamy, Network Routing: Algorithms, Protocols, and Architectures, Elsevier, 2007.

[31] X. Liu, S. Mohanraj, M. Pioro, D. Medhi, Multipath routing from a traffic engineering perspective: How beneficial is it?, in: Proceedings of the 2014 IEEE 22nd International Conference on Network Protocols (ICNP), 2014, pp. $143-154$. 
[32] D. Medhi, R. Khurana, Optimization and performance of restoration schemes for wide-area teletraffic networks, Journal of Network and Systems Management 3 (3) (1995) 265-294.

[33] C. Magnien, M. Latapy, J.-L. Guillaume, Impact of random failures and attacks on poisson and power-law random networks, ACM Computing Surveys 43 (3) (2011) 13:1-13:31.

[34] D. Medhi, Network restoration, in: M. G. C. Resende, P. M. Pardalos (Eds.), Handbook of Optimization in Telecommications, Springer US, New York, NY, USA, 2006, pp. 801-836.

[35] S. De Maesschalck, D. Colle, A. Groebbens, C. Develder, U. Lievens, P. Lagasse, M. Pickavet, P. Demeester, F. Saluta, M. Quagliatti, Intelligent optical networking for multilayer survivability, IEEE Communications Magazine 40 (1) (2002) 42-49.

[36] D. Staessens, L. Depré, D. Colle, I. Lievens, M. Pickavet, P. Demeester, A quantitative comparison of some resilience mechanisms in a multidomain ip-over-optical network environment, in: IEEE International Conference on Communications, 2006 (ICC'06), Vol. 6, 2006, pp. 2512-2517.

[37] M. Kodialam, T. Lakshman, J. B. Orlin, S. Sengupta, Preconfiguring ipover-optical networks to handle router failures and unpredictable traffic, IEEE Journal on Selected Areas in Communications 25 (5) (2007) 934948.

[38] J.-F. Labourdette, E. Bouillet, S. Chaudhuri, Role of optical network and spare router strategy in resilient ip backbone architecture, in: Proceedings. Fourth International Workshop on Design of Reliable Communication Networks, 2003 (DRCN 2003), 2003, pp. 244-253.

[39] L. Sahasrabuddhe, S. Ramamurthy, B. Mukherjee, Fault management in ipover-wdm networks: Wdm protection versus ip restoration, IEEE Journal on Selected Areas in Communications 20 (1) (2002) 21-33.

[40] I. Katib, D. Medhi, Network protection design models, a heuristic, and a study for concurrent single-link per layer failures in three-layer networks, Computer Communications (0) (2012) -.

URL http://www.sciencedirect.com/science/article/pii/ S0140366412003209

[41] S. Huang, C. Martel, B. Mukherjee, Survivable multipath provisioning with differential delay constraint in telecom mesh networks, IEEE/ACM Transactions on Networking 19 (3) (2011) 657-669.

[42] W. Ni, C. Huang, J. Wu, M. Savoie, Availability of survivable valiant load balancing (vlb) networks over optical networks, Optical Switching and Networking 10 (3) (2013) 274-289. 
[43] W. Ni, C. Huang, J. Wu, Provisioning high-availability datacenter networks for full bandwidth communication, Computer Networks 68 (0) (2014) 7194 .

[44] Y. Chen, J. Li, T. Wo, C. Hu, W. Liu, Resilient virtual network service provision in network virtualization environments, in: Proceedings of the IEEE 16th International Conference on Parallel and Distributed Systems, IEEE ICPADS, Shanghai, China, 2010, pp. 51-58.

[45] I. B. Barla, D. A. Schupke, G. Carle, Resilient virtual network design for end-to-end cloud services, in: R. Bestak, L. Kencl, L. E. Li, J. Widmer, H. Yin (Eds.), NETWORKING 2012, 11th International IFIP TC 6 Networking Conference, Vol. 7289 of Lecture Notes in Computer Science, Springer Berlin/Heidelberg, Berlin, Germany, 2012, pp. 161-174.

[46] R. Oliveira, D. Marcon, L. Bays, M. Neves, L. Buriol, L. Gaspary, M. Barcellos, No more backups: Toward efficient embedding of survivable virtual networks, in: Communications (ICC), 2013 IEEE International Conference on, 2013, pp. 2128-2132.
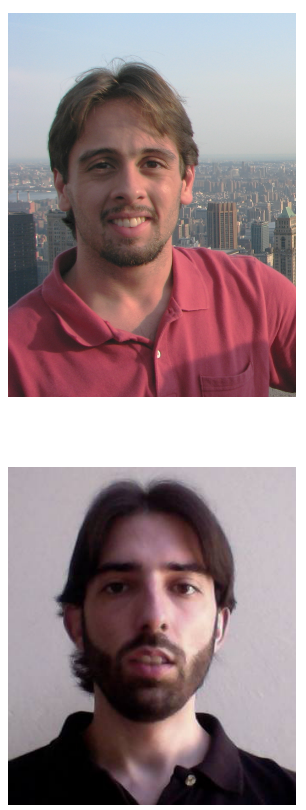

Rodrigo Ruas Oliveira is a Ph.D. student at the Institute of Informatics of the Federal University of Rio Grande do Sul (UFRGS), Brazil. He holds a B.Eng. degree in Computer Engineering from the Federal University of Rio Grande (FURG, 2010), and a M.Sc. degree in Computer Science from the Federal University of Rio Grande do Sul (UFRGS, 2012). His research interests include network virtualization, optimization, and softwaredefined networking. More information can be found at http://www.inf.ufrgs.br/ ruasoliveira.

Daniel Stefani Marcon is a Ph.D. student at the Institute of Informatics of Federal University of Rio Grande do Sul, Brazil. He holds a B.Sc. degree in Computer Science from University of Vale do Rio dos Sinos (2011) and a M.Sc. degree in Computer Science from Federal University of Rio Grande do Sul (2013). His research interests include datacenter networks, cloud computing, network virtualization and software-defined networking. See http://inf .ufrgs.br/ dsmarcon for further details. 


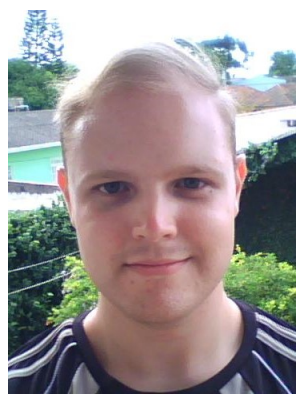

Leonardo Richter Bays is a Ph.D. student in Computer Science at the Federal University of Rio Grande do Sul (UFRGS). He received an M.Sc. degree from the same university in 2013. He is currently doing research in the areas of Network Virtualization and Software-Defined Networking (advised by Professor Luciano Paschoal Gaspary), and has worked as a collaborator in research projects such as SecFuNet (Security for Future Networks) and ReVir (Virtual Networks on Future Internet). More information can be found at http://www.inf .ufrgs.br/ lrbays/.

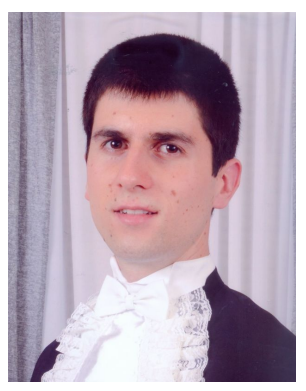

Miguel Cardoso Neves is a final year B.Eng. student at the Institute of Informatics of the Federal University of Rio Grande do Sul (UFRGS), Brazil. His research interests include network virtualization, optimization, data center networking, and software-defined networking. More information can be found at http:// lattes.cnpq.br/1395001866815977.

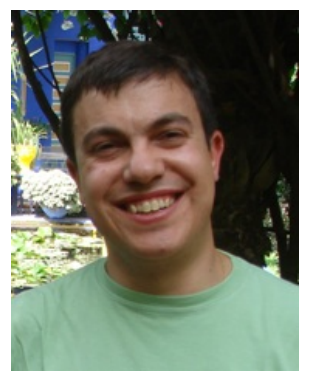

Luciano Paschoal Gaspary holds a Ph.D. in Computer Science (UFRGS, 2002) and is an Associate Professor at the Institute of Informatics, UFRGS. Prof. Gaspary has been involved in various research areas, mainly computer networks, network management and computer system security. He is author of more than 120 full papers published in leading peer-reviewed publications and has a history of dedication to research activities such as organization of scientific events, participation in the TPC of relevant symposia, and participation as editorial board member of various journals. His research interests include network, service, and application management, network virtualization, software-defined networking, cloud computing, security of large-scale distributed systems (e.g., P2P), IT service management, and intrusion detection and prevention. More information can be found at http://lattes. cnpq.br/3059640410928425. 


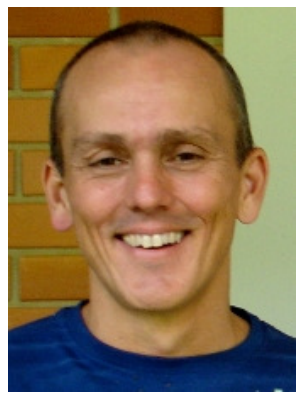

Marinho P. Barcellos is an Associate Professor in the Institute of Informatics at Federal University of Rio Grande do Sul (UFRGS), Brazil. He received B.Sc. and M.Sc. degrees in Computer Science from UFRGS (1989 and 1993, respectively) and Ph.D. degree in Computer Science from University of Newcastle Upon Tyne (1998). Prior to joining UFRGS in 2008, Prof. Barcellos has worked at UNISINOS and later at PUC-RS. In 2003-2004, he worked for University of Manchester (with British Telecomm research labs). He has authored many papers in leading journals and conferences related to computer networks, network and service management, and computer security, also serving as TPC member and chair, including the role of General Co-Chair of ACM SIGCOMM 2016. He has authored book chapters and invited talks. Prof. Barcellos was the elected chair of the Special Interest Group on Computer Security of the Brazilian Computer Society (CESeg/SBC) 2011-2012. He is a member of SBC, IEEE and ACM. His current research interests are security \& performance of cloud computing data center networks, software-defined networking, and information-centric networks. More information can be found at http://www.inf.ufrgs.br/ marinho.

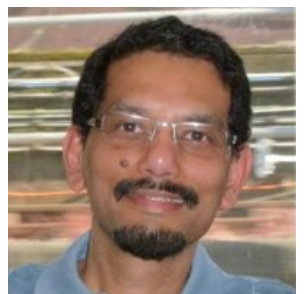

Deep Medhi is a Curators' Professor in the Department of Computer Science \& Electrical Engineering at the University of Missouri-Kansas City, USA, and a honorary professor in the Department of Computer Science \& Engineering at the Indian Institute of Technology-Guwahati, India. He received B.Sc. in Mathematics from Cotton College, Gauhati University, India, M.Sc. in Mathematics from the University of Delhi, India, and his Ph.D. in Computer Sciences from the University of Wisconsin-Madison, USA. Prior to joining UMKC in 1989, he was a member of the technical staff at AT\&T Bell Laboratories. He served as an invited visiting professor at the Technical University of Denmark, a visiting research fellow at Lund Institute of Technology, Sweden, and State University of Campinas, Brazil. As a Fulbright Senior Specialist, he was a visitor at Bilkent University, Turkey, and Kurukshetra University, India. He is the Editor-in-Chief of Springer's Journal of Network and Systems Management, and is on the editorial board of IEEE/ACM Transactions on Networking, IEEE Transactions on Network and Service Management, and IEEE Communications Surveys \& Tutorials. He has published over 125 papers, and is co-author of the books Routing, Flow, and Capacity Design in Communication and Computer Networks (2004) and Network Routing: Algorithms, Protocols, and Architectures (2007), both published by Morgan Kaufmann Publishers, an imprint of Elsevier Science. His research interests are multi-layer networking, network virtualization, data center optimization, and network routing, design, and survivability. His research has been funded by NSF, DARPA, and industries. 